\title{
MONGE SOLUTIONS FOR DISCONTINUOUS HAMILTONIANS
}

\author{
Ariela Briani ${ }^{1}$ And Andrea Davini ${ }^{1}$
}

\begin{abstract}
We consider an Hamilton-Jacobi equation of the form

$$
H(x, D u)=0 \quad x \in \Omega \subset \mathbb{R}^{N},
$$

where $H(x, p)$ is assumed Borel measurable and quasi-convex in $p$. The notion of Monge solution, introduced by Newcomb and $\mathrm{Su}$, is adapted to this setting making use of suitable metric devices. We establish the comparison principle for Monge sub and supersolution, existence and uniqueness for equation (1) coupled with Dirichlet boundary conditions, and a stability result. The relation among Monge and Lipschitz subsolutions is also discussed.
\end{abstract}

Mathematics Subject Classification. 49J25, 35C15, 35R05.

Received January 15, 2004.

\section{INTRODUCTION}

We consider the Hamilton-Jacobi equation

$$
H(x, D u)=0 \quad x \in \Omega \subset \mathbb{R}^{N},
$$

where $D u$ is the gradient of the unknown function $u: \Omega \rightarrow \mathbb{R}$ and $H: \bar{\Omega} \times \mathbb{R}^{N} \rightarrow \mathbb{R}$ is the Hamiltonian. We are concerned with the study of equation (2) in the framework of discontinuous Hamiltonians: indeed, $H$ will be assumed to be only Borel measurable, and quasi-convex in the $p$-variable for every $x \in \bar{\Omega}$. The interest of this issue is easily motivated by the applications: Hamilton-Jacobi equations with discontinuous ingredients arise naturally in several models, as, for example, propagation of fronts in non-homogeneous media, geometric optics in presence of layers, shape-from-shading problems.

One of the main theory concerning Hamilton-Jacobi equations is that of viscosity solutions, developed in the last twenty years. The literature on this subject is wide, as main reference we recall the books $[2,3,18]$, and the references therein.

With regard to the discontinuous case, measurable fully nonlinear equations of second order have been studied in [7], however the techniques exploited there are based on the strong maximum principle so they do not apply to first order equations.

The first order case has been less studied; we recall, among others (see e.g. $[4,17]$ ), $[8,20]$. In the first one Camilli and Siconolfi study equation (2) and give a notion of viscosity solution making use of suitable measure-theoretic devices. They prove a comparison result, and consequently, when equation (2) is coupled

\footnotetext{
Keywords and phrases. Viscosity solution, lax formula, Finsler metric.

1 Dipartimento di Matematica, Università di Pisa Lago B. Pontecorvo 5, 56127 Pisa, Italy; briani@mail.dm.unipi.it; davini@dm.unipi.it
} 
with a boundary datum, they get unicity of the solution and an integral representation formula, generalizing the one valid for the continuous case. Moreover, such a solution is proven to be the maximal among Lipschitz subsolutions, in analogy with the classical setting.

In [20], Soravia studies the following Hamilton-Jacobi equation related to optimal control problems

$$
\lambda u(x)+\sup _{a \in A}\{-f(x, a) D u(x)-h(x, a)\}=g(x)
$$

where $g$ is only Borel measurable. The viscosity solutions are defined by taking the lower and upper semicontinuous envelopes of $g$ following [16]. Uniqueness and stability results are given.

Both the recalled works start by comparing their definitions with a slightly different one, given by Newcomb and $\mathrm{Su}$ in [19]. The authors studied the equation of eikonal type

$$
H(D u)=n(x)
$$

where the discontinuity is in $n$ only, which is assumed to be lower semicontinuous. They introduce the definition of Monge solution, which is shown to be consistent with the viscosity notion when $n$ is continuous. In this framework they establish the comparison principle for sub and supersolutions, existence and uniqueness results for (3) with Dirichlet boundary conditions, and a stability result.

In this paper we want to extend this definition to equations of the more general form (2) and to generalize to this case the above-mentioned results. In order to be more precise about the type of discontinuities we admit, let us specify that we will deal with Borel-measurable Hamiltonians $H$ such that $Z(x):=\left\{p \in \mathbb{R}^{N} \mid H(x, p) \leq 0\right\}$ is closed and convex and $\partial Z(x)=\left\{p \in \mathbb{R}^{N} \mid H(x, p)=0\right\}$ for every $x \in \bar{\Omega}$. Moreover, we assume that there exist two positive constants $\alpha$ and $\beta$ such that $B_{\alpha}(0) \subset Z(x) \subset B_{\beta}(0)$ for every $x \in \bar{\Omega}$.

In analogy with [19], we need to recall that the optical length function relative to the Hamiltonian $H$ is the map $S: \bar{\Omega} \times \bar{\Omega} \rightarrow \mathbb{R}$ defined as follows:

$$
S(x, y):=\inf \left\{\int_{0}^{1} \sigma(\gamma(t), \dot{\gamma}(t)) \mathrm{d} t \mid \gamma \in \operatorname{Lip}([0,1], \bar{\Omega}), \gamma(0)=x, \gamma(1)=y\right\}
$$

for every $x, y \in \bar{\Omega}$, where $\sigma$ is the support function of the section $Z(x)$, namely $\sigma(x, \xi):=\sup \{\langle-\xi, p\rangle \mid p \in Z(x)\}$. Given $u \in C(\Omega)$, we say that $u$ is a Monge solution (resp. subsolution, supersolution) of (2) in $\Omega$ if for each $x_{0} \in \Omega$ there holds

$$
\liminf _{x \rightarrow x_{0}} \frac{u(x)-u\left(x_{0}\right)+S\left(x_{0}, x\right)}{\left|x-x_{0}\right|}=0 \quad(\text { resp. } \geq, \leq) .
$$

As it should be clear by the above definition, the properties of Monge sub and supersolutions strictly depend on those enjoyed by the optical length function $S$. As we will see, the function $S$ is a geodesic, non-symmetric distance, which corresponds, with the notations of Section 2, to $d_{\sigma}$ (defined by (10)). Therefore, as a preliminary step, we collect and prove some results about non-symmetric distances (see Sects. 2 and 3). Those results are then specialized to $S$ to carry on the study of Monge solutions. In this regard, we underline that the semicontinuity of the function $n$ in (3) is mainly used in [19] to obtain semicontinuity of the length functional $\mathbb{L}_{\sigma}$ (defined by (11) in Sect. 2), and therefore the existence of an optimal path for $S(x, y)$, namely a path of minimal $\mathbb{L}_{\sigma}$-length. This technical difficulty is overcome here by introducing the metric length of a curve with respect to the non-symmetric distance $S$ ( $c f$. formula (8) in Sect. 2), which is the relaxed functional of $\mathbb{L}_{\sigma}$. The existence of a minimal path (with respect to the metric $S$-length) for $S(x, y)$ for all $x, y \in \bar{\Omega}$ is then an easy consequence of the results of Section 2. Consequently, under the above-stated conditions for the Hamiltonian, we obtain a comparison result among Monge sub and supersolutions of equation (2) (Th. 5.1). This implies moreover that, under certain compatibility conditions for the boundary data, the Dirichlet problem

$$
\begin{cases}H(x, D u)=0 & \text { in } \Omega \\ u=g & \text { on } \partial \Omega\end{cases}
$$


has a unique Monge solution $u$, given by Lax formula

$$
u(x):=\inf _{y \in \partial \Omega}\{S(x, y)+g(y)\} \quad \text { for all } x \in \bar{\Omega}
$$

thus recovering a well known result in the case of a continuous Hamiltonian.

In the continuous case, moreover, the function defined by (6) is also the maximal element in the class of Lipschitz subsolutions of (5). As already remarked in [19,20], this is no longer true in general when dealing with Monge solutions of discontinuous Hamilton-Jacobi equations. However, when the Hamiltonian is mildly discontinuous, the previous maximality property still holds. This issue will be investigated in a more detailed way in Section 7 ( $c f$. Th. 7.3). As a matter of fact, this will be done by comparing the definition of Monge solution adopted here with that of viscosity solution introduced by Camilli and Siconolfi in [8]. The main difference between the two approaches relies upon the definition of optical length function: while here $S$ is defined by (4) through an infimum, the corresponding function $L^{\Omega}$ in [8] is defined through a sup-inf process ( $c f$. Sect. 7 for the definition). The latter has the effect of rendering the function $L^{\Omega}$ independent of modifications of the Hamiltonian $H$ (and consequently of the support function $\sigma$ ) on negligible subset of $\bar{\Omega}$ with respect to the $x$-variable, a property which is necessary if one is interested in keeping the equivalence (holding in the continuous setting, see [2]) between Lipschitz and viscosity subsolutions of (2). This in particular gives the maximality of the viscosity solution of (5) among Lipschitz subsolutions (cf. [8], Prop. 3.6). Some problems arise instead when one deals with sequences of solutions: in [8], Example 7.2, the authors consider a sequence of continuous Hamilton-Jacobi equations converging to a limit equation for which it is easy to exhibit a corresponding sequence of viscosity solutions (in the classical sense) uniformly converging to a function which is not the viscosity solution, in the sense there considered, of the limit equation (actually, it turns out to be a Monge solution, see Ex. 6.5). The main reason of this behavior is that the family of distances that can be obtained through such a sup-inf process is not closed for the uniform convergence ( $c f$. Prop. 3.7).

On the other hand, the definition of optical length function given here strictly depends on the pointwise behavior of the Hamiltonian and changing it in the $x$-variable over negligible sets does count. Moreover, the class of distances obtained through (4) is closed for the uniform convergence (in fact, it is compact, cf. Sect. 2 and Th. 2.6). In particular, with this approach one can treat optimization problems such as

$$
\min \left\{\int_{\Omega}\left|u_{a}-f\right|^{2} \mathrm{~d} x \mid a: \bar{\Omega} \rightarrow[\alpha, \beta] \text { Borel measurable, } \int_{\Omega} a(x) \mathrm{d} x \leq m\right\},
$$

where $\alpha, \beta$ and $m$ are suitable positive constants, $f: \Omega \rightarrow \mathbb{R}$ is a given function and $u_{a}$ is the Monge solution of the following equation, depending on the control $a$ :

$$
\begin{cases}|D u|=a(x) & \text { in } \Omega \\ u=0 & \text { on } \partial \Omega\end{cases}
$$

Indeed, the problem can be attacked using the direct method of the Calculus of Variations: chosen a minimizing sequence $\left(a_{n}\right)_{n}$, it is easy to see, using the representation formula (6) and the recalled compactness result (Th. 2.6), that the corresponding solutions $u_{a_{n}}$ converge uniformly to a function $u$. To show that $u$ is the Monge solution of problem (7) for an admissible control $a$ one can refer to the results proved in [10] (specifically, Ths. 4.3 and 4.7, cf. also Ex. 8.2).

Our paper is organized as follows. In Section 2 we recall the main results concerning non-symmetric distances. The study of the properties of distances is carried on in Section 3. In particular, we compare two different ways of deriving a distance from a function $\varphi \in \mathcal{M}$, namely (10) and (17), and we will examine under which conditions they are equivalent. The properties derived in the general framework of geodesic distances are applied in Section 4 to the optical length function $S$, and some properties of Monge sub and supersolutions are deduced. In particular, we show that the definition of Monge solution reduces to the viscosity one when the Hamiltonian is continuous. Section 5 contains the proofs of the comparison principle (Th. 5.1) and the 
solvability of the Dirichlet problem (5) (Th. 5.3). In Section 6 a stability result is proven under a suitable convergence of Hamiltonians, which includes, as special cases, the ones more classically considered, such as uniform convergence. In Section 7 we discuss the pointwise behavior of Monge solutions of problem (5) and the relation among Monge and Lipschitz subsolutions. The paper is ended with some examples. In particular, we will show how Monge solutions of certain eikonal equations arise naturally as asymptotic limit of viscosity solutions of evolutive Hamilton-Jacobi equations with continuous ingredients.

Notation. We write here a list of symbols used throughout this paper.

$\begin{array}{ll}\mathbb{S}^{N-1} & (N-1) \text {-dimensional unitary sphere of } \mathbb{R}^{N} \\ B_{r}(x) & \text { open ball in } \mathbb{R}^{N} \text { of radius } r \text { centred in } x \\ I & \text { closed interval }[0,1] \\ \mathcal{L}^{k} & k \text {-dimensional Lebesgue measure } \\ \mathcal{H}^{k} & k \text {-dimensional Hausdorff measure } \\ |x| & \text { Euclidean norm of the vector } x \in \mathbb{R}^{N} \\ \mathbb{R}_{+} & \text {non-negative real numbers } \\ \chi_{E} & \text { the characteristic function of the set } E .\end{array}$

In this paper $N$ will denote an integer number, $\alpha$ and $\beta$ two positive constants with $\beta>\alpha$, and $\Omega$ a bounded domain (i.e. an open connected set) of $\mathbb{R}^{N}$ with Lipschitz boundary. A subset of $\mathbb{R}^{N}$ is said to be negligible if its $N$-dimensional Lebesgue measure is null. With the word curve or path we will always indicate a Lipschitz function from the interval $I:=[0,1]$ to $\bar{\Omega}$. Any curve $\gamma$ is always supposed to be parameterized by constant speed, i.e. in such a way that $|\dot{\gamma}(t)|$ is constant for $\mathcal{L}^{1}$-a.e. $t \in I$. We will say that a sequence of curves $\left(\gamma_{n}\right)_{n}$ (uniformly) converges to a curve $\gamma$ to mean that $\sup _{t \in I}\left|\gamma_{n}(t)-\gamma(t)\right|$ tends to zero as $n$ goes to infinity. We will denote by $\operatorname{Lip}_{x, y}$ the family of curves $\gamma$ which join $x$ and $y$, i.e. such that $\gamma(0)=x$ and $\gamma(1)=y$. Last, for a measurable function $f: I \rightarrow \mathbb{R}^{N},\|f\|_{\infty}$ stands for $\sqrt{\sum_{i=0}^{N}\left\|f_{i}\right\|_{L^{\infty}(I)}^{2}}$, where $f_{i}$ and $\left\|f_{i}\right\|_{L^{\infty}(I)}$ denotes the $i$-th component of $f$ and the $\mathrm{L}^{\infty}$-norm of $f_{i}$ respectively.

\section{Preliminaries on Geodesic Distances}

In this section we will describe the main definitions and properties of Finsler distances that will be useful to study the optical length functions $S$ and consequently the properties of Monge solutions. In the sequel, a distance $d$ on $\bar{\Omega}$ will be called non-symmetric if the identity $d(x, y)=d(y, x)$ may fail to hold on $\bar{\Omega} \times \bar{\Omega}$.

We stress that definitions and results stated in this section are essentially known, but usually given in literature considering symmetric distances. Proofs can be easily adapted to our setting by minor changes, and will therefore omitted ( $c f$. [11]).

First, let us define the classical $d$-length of $\gamma$, obtained as the supremum of the $d$-lengths of inscribed polygonal curves:

$$
\mathrm{L}_{d}(\gamma):=\sup \left\{\sum_{i=0}^{m-1} d\left(\gamma\left(t_{i}\right), \gamma\left(t_{i+1}\right)\right) \mid 0=t_{0}<t_{1}<\ldots<t_{m}=1, m \in \mathbb{N}\right\} .
$$

We will say that $d$ is a geodesic distance if it satisfies the following identity:

$$
d(x, y)=\inf \left\{\mathrm{L}_{d}(\gamma) \mid \gamma \in \operatorname{Lip}_{x, y}\right\} \quad \text { for every }(x, y) \in \bar{\Omega} \times \bar{\Omega} .
$$

All distances considered in this paper will fulfill the following hypotheses:

(d1) $d$ is non-symmetric;

(d2) $d$ is geodesic; 
(d3) there exist two positive constants $\alpha$ and $\beta$, such that

$$
\alpha|x-y| \leq d(x, y) \leq \beta|x-y| \quad \text { locally in } \Omega
$$

(i.e. for every $x_{0} \in \Omega$ there exists an open ball $B_{r}\left(x_{0}\right) \subset \Omega$ such that the above inequality holds for every $\left.x, y \in B_{r}\left(x_{0}\right)\right)$.

Any distance $d$ which satisfies the Hypotheses $(\mathrm{d} 1)-(\mathrm{d} 3)$ induces on $\bar{\Omega}$ a topology which is equivalent to the Euclidean one. In particular, by applying to our framework a classical theorem due to Busemann (cf. [1], Th. 4.3.1), we obtain what follows.

Proposition 2.1. The length functional $\mathrm{L}_{d}$ is lower semicontinuous with respect to the uniform convergence of paths, namely if $\left(\gamma_{n}\right)_{n}$ converges to $\gamma$ then $\mathrm{L}_{d}(\gamma) \leq \liminf _{n} \mathrm{~L}_{d}\left(\gamma_{n}\right)$. In particular, for every couple of points $x, y$ in $\bar{\Omega}$ there exists a curve $\gamma \in \operatorname{Lip}_{x, y}$ which is a path of minimal d-length, i.e. such that $\mathrm{L}_{d}(\gamma)=d(x, y)$.

A Borel-measurable function $\varphi: \bar{\Omega} \times \mathbb{R}^{N} \rightarrow \mathbb{R}_{+}$will be said to be a (weak) Finsler metric on $\bar{\Omega}$ if $\varphi(x, \cdot)$ is 1-homogeneous for every $x \in \bar{\Omega}$ and convex for $\mathcal{L}^{N}$-a.e. $x \in \bar{\Omega}$.

We now fix two positive constants $\alpha$ and $\beta$ and we consider the following family of functions:

$$
\mathcal{M}:=\left\{\varphi \text { Finsler metrics on } \bar{\Omega}: \alpha|\xi| \leq \varphi(x, \xi) \leq \beta|\xi| \text { on } \bar{\Omega} \times \mathbb{R}^{N}\right\}
$$

For each $\varphi \in \mathcal{M}$, we can define a function $d_{\varphi}$ on $\bar{\Omega} \times \bar{\Omega}$ through the formula

$$
d_{\varphi}(x, y):=\inf \left\{\mathbb{L}_{\varphi}(\gamma) \mid \gamma \in \operatorname{Lip}_{x, y}\right\}
$$

where the length functional $\mathbb{L}_{\varphi}$ is defined by

$$
\mathbb{L}_{\varphi}(\gamma):=\int_{0}^{1} \varphi(\gamma(t), \dot{\gamma}(t)) \mathrm{d} t
$$

The main properties of $d_{\varphi}$ are summarized below.

Proposition 2.2. The function $d_{\varphi}(x, y)$ given by (10) is well defined on $\bar{\Omega} \times \bar{\Omega}$ and satisfies the following properties:

(i) $0 \leq d_{\varphi}(x, y) \leq d_{\varphi}(x, z)+d_{\varphi}(z, y)$ for all $x, y, z \in \bar{\Omega}$;

(ii) $\alpha|x-y| \leq d_{\varphi}(x, y) \leq \beta|x-y|$ locally in $\Omega$;

(iii) $d_{\varphi}$ is Lipschitz on $\bar{\Omega} \times \bar{\Omega}$, with Lipschitz constant equal to $2 \beta C$, where $C \geq 1$ is the Lipschitz constant of $\partial \Omega$.

In particular, $d_{\varphi}$ is a non-symmetric distance, locally equivalent to the Euclidean one.

Proof. Let $\gamma$ be a curve. Since the map $t \mapsto(\gamma(t), \dot{\gamma}(t))$ is Lebesgue measurable on $I$, and $\varphi$ is Borel measurable on $\bar{\Omega} \times \mathbb{R}^{N}$, their composition $\varphi(\gamma(t), \dot{\gamma}(t))$ is Lebesgue measurable on $I$. Therefore the integral in (11) is well defined and so is $d_{\varphi}$. The remainder of the claim is a simple consequence of the definitions.

Remark 2.3. With regard to item (ii) in the statement of Proposition 2.2, it is worth noticing that the inequality $d_{\varphi}(x, y) \geq \alpha|x-y|$ actually holds for every $x, y \in \bar{\Omega}$, while $d_{\varphi}(x, y) \leq \beta|x-y|$ holds true whenever the Euclidean segment joining $x$ to $y$ lies in $\bar{\Omega}$.

Next proposition clarifies the relation between the functional (11) and the (intrinsic) metric length functional (8) (cf. [12], Th. 4.3).

Proposition 2.4. Let $d:=d_{\varphi}$ with $\varphi \in \mathcal{M}$. Then for any $\gamma \in \operatorname{Lip}(I, \bar{\Omega})$ we have:

$$
\mathrm{L}_{d}(\gamma)=\inf \left\{\liminf _{n \rightarrow+\infty} \mathbb{L}_{\varphi}\left(\gamma_{n}\right):\left(\gamma_{n}\right)_{n} \text { converges to } \gamma \text { in } \operatorname{Lip}(I, \bar{\Omega})\right\}
$$


namely $\mathrm{L}_{d}$ is the relaxed functional of $\mathbb{L}_{\varphi}$ on $\operatorname{Lip}(I, \bar{\Omega})$. In particular, $d$ is a distance of geodesic type according to definition (9).

Remark 2.5. By Proposition 2.4, $\mathbb{L}_{\varphi}$ will coincide with $\mathrm{L}_{d}$ whenever $\mathbb{L}_{\varphi}$ is lower semicontinuous on $\operatorname{Lip}(I, \bar{\Omega})$. This happens, for instance, when $\varphi$ is lower semicontinuous on $\bar{\Omega} \times \mathbb{R}^{N}$ and $\varphi(x, \cdot)$ is convex on $\mathbb{R}^{N}$ for every $x \in \bar{\Omega}(c f .[5]$, Th. 4.1.1).

Let us denote by $\mathcal{D}$ the family of distances on $\bar{\Omega}$ generated by the metrics $\mathcal{M}$, namely $\mathcal{D}:=\left\{d_{\varphi} \mid \varphi \in \mathcal{M}\right\}$. We endow $\mathcal{D}$ with the metric given by the uniform convergence on $\bar{\Omega} \times \bar{\Omega}$. This convergence is equivalent to the $\Gamma$-convergence of the relative length functionals $\mathrm{L}_{d_{n}}$ to $\mathrm{L}_{d}$ with respect to the uniform convergence of paths, as proved in [6], Theorem 3.1. Moreover, we have the following (cf. [6], Th. 3.1):

Theorem 2.6. The set $\mathcal{D}$ is endowed with the metric given by the uniform convergence of distances on $\bar{\Omega} \times \bar{\Omega}$ is a metrizable compact space.

Next proposition describes the convergence of elements of $\mathcal{D}$ in terms of the convergence of the generating metrics. A proof is given in [11].

Proposition 2.7. Let $\varphi, \varphi_{n} \in \mathcal{M}$ and $d$ and $d_{n}$ be the distances associated respectively to $\varphi$ and $\varphi_{n}$ through (10). Then $\left(d_{n}\right)_{n}$ converges uniformly to $d$ in the following cases:

(i) $\left(\varphi_{n}\right)_{n}$ converges uniformly to $\varphi$ on compact subset of $\bar{\Omega} \times \mathbb{R}^{N}$;

(ii) $\varphi_{n}$ are lower semicontinuous in $x$, convex in $\xi$ and converge increasingly to $\varphi$ pointwise on $\bar{\Omega} \times \mathbb{R}^{N}$;

(iii) $\left(\varphi_{n}\right)_{n}$ converges decreasingly to $\varphi$ pointwise on $\bar{\Omega} \times \mathbb{R}^{N}$.

An integral representation of the $d$-length of a curve $\gamma$ can be given in terms of its metric derivative, as known by classical results on metric spaces [1], and this result can be easily extended to the non-symmetric setting (see [11]). In particular, when the curve $\gamma$ lies in $\Omega$ (i.e. $\gamma(I) \subset \Omega$ ), the following holds:

$$
\mathrm{L}_{d}(\gamma)=\int_{0}^{1} \varphi_{d}(\gamma(t), \dot{\gamma}(t)) \mathrm{d} t
$$

i.e. $\mathrm{L}_{d}(\gamma)=\mathbb{L}_{\varphi_{d}}(\gamma)$ (cf. $[11,15]$, Th. 2.5), where $\varphi_{d}$ is the Finsler metric on $\Omega$ associated to $d$ by derivation, given by

$$
\varphi_{d}(x, \xi):=\limsup _{t \rightarrow 0^{+}} \frac{d(x, x+t \xi)}{t} \quad(x, \xi) \in \Omega \times \mathbb{R}^{N} .
$$

Definition (14) might be suitably extended to the boundary of $\Omega$ ( $c f$. [11]). This generalization is not needed here and will be not detailed any further. We summarize in the next proposition the main properties of $\varphi_{d}$. For the proof, we refer to $[13,15]$.

Proposition 2.8. The function $\varphi_{d}: \Omega \times \mathbb{R}^{N} \rightarrow \mathbb{R}_{+}$given in (14) is Borel-measurable. Moreover we have:

(i) $\varphi_{d}(x, \cdot)$ is positively 1-homogeneous for every $x \in \Omega$;

(ii) $\left|\varphi_{d}(x, \xi)-\varphi_{d}(x, \nu)\right| \leq \beta|\xi-\nu|$ for every $x \in \Omega$ and every $\xi, \nu \in \mathbb{R}^{N}$;

(iii) $\varphi_{d}(x, \cdot)$ is convex for $\mathcal{L}^{N}$-a.e. $x \in \Omega$.

To sum up, any function $\varphi \in \mathcal{M}$ gives rise to a distance $d:=d_{\varphi}$ in $\mathcal{D}$ through (10). To such a distance $d$, one can associate by derivation the Finsler metric $\varphi_{d}$ given by (14). Even if $\varphi_{d}$ need not be equal to $\varphi$, some relations between them can be deduced. 
Proposition 2.9. Let $\varphi \in \mathcal{M}$ and $d:=d_{\varphi}$ be the non-symmetric distance associated to $\varphi$ according to (10). Then there exists a negligible set $N \subset \Omega$ such that

$$
\varphi_{d}(x, \xi) \leq \varphi(x, \xi) \quad \text { for every }(x, \xi) \in \Omega \backslash N \times \mathbb{R}^{N}
$$

where $\varphi_{d}$ is defined in (14). Moreover we have:

(i) if $\varphi(x, \cdot)$ is convex on $\mathbb{R}^{N}$ for every $x \in \Omega$ and $\varphi(\cdot, \xi)$ is lower semicontinuous for every $\xi \in \mathbb{R}^{N}$, we have

$$
\varphi_{d}(x, \xi) \geq \liminf _{t \rightarrow 0^{+}} \frac{d(x, x+t \xi)}{t} \geq \varphi(x, \xi) \quad \text { for every }(x, \xi) \in \Omega \times \mathbb{R}^{N}
$$

In particular, $\varphi_{d}(x, \xi)=\varphi(x, \xi)$ on $\Omega \backslash N \times \mathbb{R}^{N}$

(ii) if $\varphi(\cdot, \xi)$ is upper semicontinuous for every $\xi \in \mathbb{R}^{N}$, we have $\varphi_{d}(x, \xi) \leq \varphi(x, \xi)$ for every $(x, \xi) \in \Omega \times \mathbb{R}^{N}$.

Proof. Let us fix a vector $\xi \in \mathbb{S}^{N-1}$ and, for every $x_{0} \in \Omega$, let us define the curve $\gamma_{x_{0}}(s):=x_{0}+s \xi$. Let $t$ be a Lebesgue point for the map $s \mapsto \varphi\left(\gamma_{x_{0}}(s), \xi\right)$. For $h>0$ small enough we have

$$
\frac{1}{h} \int_{t}^{t+h} \varphi\left(\gamma_{x_{0}}(s), \xi\right) \mathrm{d} s=\frac{1}{h} \int_{0}^{1} \varphi\left(\gamma_{x_{0}}(t+h \tau), h \xi\right) \mathrm{d} \tau \geq \frac{d\left(\gamma_{x_{0}}(t), \gamma_{x_{0}}(t)+h \xi\right)}{h}
$$

so, by taking the limsup as $h \rightarrow 0^{+}$, we get $\varphi_{d}\left(\gamma_{x_{0}}(t), \xi\right) \leq \varphi\left(\gamma_{x_{0}}(t), \xi\right)$. Since $\mathcal{L}^{1}-$ a.e. $t \in \mathbb{R}$ is a Lebegue point for $\varphi\left(\gamma_{x_{0}}(\cdot), \xi\right)$ and $x_{0}$ was arbitrarily chosen in $\Omega$, Fubini's Theorem implies that $\varphi_{d}(x, \xi) \leq \varphi(x, \xi)$ for $\mathcal{L}^{N}$-a.e. $x \in \Omega$. Then we can take a dense sequence $\left(\xi_{n}\right)_{n}$ in $\mathbb{S}^{N-1}$ and repeat the above argument for each $\xi_{n}$. Recalling that the functions $\varphi_{d}(x, \cdot)$ and $\varphi(x, \cdot)$ are continuous (and 1-homogeneous) for $\mathcal{L}^{N}-a . e$. $x \in \Omega$, we get, by the density of $\left(\xi_{n}\right)_{n}$, that $\varphi_{d}(x, \xi) \leq \varphi(x, \xi)$ for $\mathcal{L}^{N}$-a.e. $x \in \Omega$ and for every $\xi \in \mathbb{R}^{N}$.

(i) Let us assume $\varphi$ lower semicontinuous in $x$ and convex in $\xi$ and fix $(x, \xi) \in \Omega \times \mathbb{S}^{N-1}$. By lower semicontinuity, for every $\varepsilon>0$ there exists $r=r(\varepsilon, x)>0$ such that $B_{r}(x) \subset \Omega$ and $\varphi(y, \xi)>\varphi(x, \xi)-\varepsilon$ for every $y \in B_{r}(x)$. Moreover, by the Lipschitz continuity of $\varphi$ in $\xi$ and by possibly choosing a smaller $r$, the previous inequality holds in $B_{r}(x) \times B_{r}(\xi)$. Hence, as $\mathbb{S}^{N-1}$ is compact, there exists a suitable $r>0$ such that

$$
\varphi(y, \xi) \geq \varphi(x, \xi)-\varepsilon \quad \text { for every }(y, \xi) \in B_{r}(x) \times \mathbb{S}^{N-1} .
$$

Choose a $d$-minimizing sequence of paths $\left(\gamma_{n}\right)_{n} \subset \operatorname{Lip}_{x, x+t \xi}$. For $t$ small enough, the curves $\gamma_{n}$ lie within $B_{r}(x)$. Then, for $n$ big enough, we have

$$
\mathbb{L}_{\varphi}\left(\gamma_{n}\right)=\int_{0}^{1} \varphi\left(\gamma_{n}(s), \dot{\gamma}_{n}(s)\right) \mathrm{d} s \geq \int_{0}^{1}\left(\varphi\left(x, \dot{\gamma}_{n}(s)\right)-\varepsilon\left|\dot{\gamma}_{n}(s)\right|\right) \mathrm{d} s \geq t\left(\varphi(x, \xi)-2 \frac{\beta}{\alpha} \varepsilon\right),
$$

where for the last estimate we have used Jensen's inequality applied to the convex function $\varphi(x, \cdot)$ and the fact that $\alpha \int_{0}^{1}\left|\dot{\gamma}_{n}\right| \mathrm{d} s \leq \mathbb{L}_{\varphi}\left(\gamma_{n}\right) \leq 2 d(x, x+t \xi) \leq 2 \beta t$ if $n$ is large enough. Letting $n$ go to $+\infty$ in the above inequality we obtain

$$
\frac{d(x, x+t \xi)}{t} \geq \varphi(x, \xi)-2 \frac{\beta}{\alpha} \varepsilon .
$$

By taking the liminf of (15) as $t \rightarrow 0^{+}$and since $\varepsilon>0, x \in \Omega$ and $\xi \in \mathbb{S}^{N-1}$ were arbitrary we obtain

$$
\varphi_{d}(x, \xi) \geq \liminf _{t \rightarrow 0^{+}} \frac{d(x, x+t \xi)}{t} \geq \varphi(x, \xi) \quad \text { for every }(x, \xi) \in \Omega \times \mathbb{S}^{N-1},
$$

and the claim follows by 1-homogeneity in $\xi$.

(ii) Fix $(x, \xi) \in \Omega \times \mathbb{S}^{N-1}$. By the upper-semicontinuity assumption, there exists an $r>0$ such that $B_{r}(x) \subset \Omega$ and $\varphi(y, \xi)<\varphi(x, \xi)+\varepsilon$ for every $y \in B_{r}(x)$. For $t$ small enough the curve $\gamma_{t}(s):=x+s(t \xi)$ lies within $B_{r}(x)$, 
so we have

and hence

$$
d(x, x+t \xi) \leq \int_{0}^{1} \varphi(x+s(t \xi), t \xi) \mathrm{d} s \leq \int_{0}^{1}(\varphi(x, t \xi)+\varepsilon t) \mathrm{d} s=t(\varphi(x, \xi)+\varepsilon)
$$

$$
\frac{d(x, x+t \xi)}{t} \leq \varphi(x, \xi)+\varepsilon .
$$

By taking the limsup in (16) as $t \rightarrow 0^{+}$and since $\varepsilon>0, x \in \Omega$ and $\xi \in \mathbb{S}^{N-1}$ were arbitrary, we obtain the claim.

From the previous proposition we deduce the following result.

Corollary 2.10. Let $\varphi \in \mathcal{M}$ and $d:=d_{\varphi}$ be the non-symmetric distance associated to $\varphi$ according to (10). If $\varphi(\cdot, \xi)$ is continuous on $\Omega$ for every $\xi \in \mathbb{R}^{N}$ and $\varphi(x, \cdot)$ is convex on $\mathbb{R}^{N}$ for every $x \in \Omega$, then $\varphi_{d}(x, \xi)=$ $\varphi(x, \xi)$ for every $(x, \xi) \in \Omega \times \mathbb{R}^{N}$.

\section{Fine properties of Distances}

For later use, we need to introduce a different way to derive a distance from an element of $\mathcal{M}$. Following [15], we introduce the notion of transversality: we say that a curve $\gamma$ is transversal to the negligible set $E$ if $\mathcal{H}^{1}(\gamma(I) \cap$ $E)=0$. Then, for each $\varphi \in \mathcal{M}$, we define a function $\widetilde{d}_{\varphi}$ on $\bar{\Omega} \times \bar{\Omega}$ through the following formula:

$$
\widetilde{d}_{\varphi}(x, y):=\sup _{\mathcal{L}^{N}(E)=0}\left\{\inf \left\{\mathbb{L}_{\varphi}(\gamma) \mid \gamma \in \operatorname{Lip}_{x, y}, \gamma \text { transversal to } E\right\}\right\}
$$

Let us denote by $\widetilde{\mathcal{D}}$ the space of distances generated by the elements of $\mathcal{M}$ through (17), namely $\widetilde{\mathcal{D}}:=\left\{\widetilde{d}_{\varphi}:\right.$ $\varphi \in \mathcal{M}\}$. Its main properties are summarized in the next theorem.

Theorem 3.1. Let $\varphi \in \mathcal{M}$ and let $\widetilde{d}_{\varphi}$ be the distance defined by (17). Then there exists a negligible set $F \subset \bar{\Omega}$ such that

$$
\widetilde{d}_{\varphi}(x, y)=\inf \left\{\mathbb{L}_{\varphi}(\gamma) \mid \gamma \in \operatorname{Lip}_{x, y}, \gamma \text { transversal to } F\right\} .
$$

Moreover, if we set $\widetilde{\varphi}(x, \xi):=\varphi(x, \xi) \chi_{\bar{\Omega} \backslash F}(x)+\beta|\xi| \chi_{F}(x)$, we have that $\widetilde{d}_{\varphi}=d_{\widetilde{\varphi}}$, where $d_{\tilde{\varphi}}$ is the distance associated to $\widetilde{\varphi}$ through (10). In particular, we have that $\widetilde{\mathcal{D}} \subset \mathcal{D}$.

In order to prove Theorem 3.1, we need a preliminary lemma.

Lemma 3.2. Let $\gamma \in \operatorname{Lip}_{x, y}$ with $x, y \in \bar{\Omega}$ and let $E$ be a negligible subset of $\bar{\Omega}$. Then for every $\varepsilon>0$ there exists a curve $\gamma_{\varepsilon} \in \operatorname{Lip}_{x, y}$ transversal to $E$ and such that $\left\|\gamma_{\varepsilon}-\gamma\right\|_{W^{1, \infty}}:=\left\|\gamma_{\varepsilon}-\gamma\right\|_{\infty}+\left\|\dot{\gamma}_{\varepsilon}-\dot{\gamma}\right\|_{\infty}<\varepsilon$.

Proof. Let $\gamma \in \operatorname{Lip}_{x, y}$ and let $g(t) \in C^{1}(I)$ be a non negative function such that $g(t)=0$ for $t=0$ and $t=1$ only (take for example $g(t):=\sin (\pi t)$ ). First, let us prove that for $\mathcal{L}^{N}$-a.e. $v \in \mathbb{R}^{N}$ the curve $\gamma_{v}(t):=\gamma(t)+v g(t)$ is transversal to the set $E$. Set $F(t, v):=\gamma(t)+v g(t)$ and let $A$ be the set of points $(t, v) \in I \times \mathbb{R}^{N}$ such that $F(t, v)$ belongs to $E$. For every fixed $t \in(0,1)$, the section $A_{t}:=\left\{v \in \mathbb{R}^{N} \mid(t, v) \in A\right\}$ has zero Lebesgue measure in $\mathbb{R}^{N}$, therefore $A$ has zero Lebesgue measure in $I \times \mathbb{R}^{N}$. This implies that for every $v \in \mathbb{R}^{N} \backslash N_{0}$ the section $A_{v}:=\{t \in I \mid(t, v) \in A\}$ is $\mathcal{L}^{1}$-negligible in $I$, where $N_{0}$ is a negligible set in $\mathbb{R}^{N}$. Therefore, since $\gamma_{v}(t)$ is Lipschitz, for every $v \in \mathbb{R}^{N} \backslash N_{0}$ the set $\gamma_{v}\left(A_{v}\right)$ is $\mathcal{H}^{1}$-negligible in $\mathbb{R}^{N}$, hence the curve $\gamma_{v}$ is transversal to $E$, as $\gamma_{v}\left(A_{v}\right)=\gamma_{v}(I) \cap E$. Remark that $\left\|\gamma_{v}-\gamma\right\|_{W^{1, \infty}}=|v|\|g\|_{W^{1, \infty}}$.

If $\gamma$ lies inside $\Omega$, then for $|v|$ small enough the curves $\gamma_{v}$ lie inside $\Omega$. The claim follows by setting $\gamma_{\varepsilon}:=\gamma_{v}$ with $v \in \mathbb{R}^{N} \backslash N_{0}$ and $|v|<\varepsilon /\|g\|_{W^{1, \infty}}$.

Otherwise, let us assume that the curve $\gamma$ touches the boundary in a point $x_{0}$. By possibly subdividing $\gamma(I)$ into small subarcs, we may suppose that the curve $\gamma$ lies in $\bar{\Omega} \cap B$, where $B$ is a ball centered in $x_{0}$. This ball can be chosen small enough in such a way that there exists a cone $C:=\left\{v \in B_{\delta}(0)|\langle v, \xi\rangle>\delta| v \mid\right\}$, with $\delta>0$ and $\xi \in \mathbb{S}^{N-1}$ suitably chosen, such that $z+C \subset \Omega$ for every $z \in \partial \Omega \cap B$. Remark that, if $v \in C$, the curve $\gamma_{v}$ 
lies inside $\Omega$. Therefore, by arguing as above, the claim is achieved by setting $\gamma_{\varepsilon}:=\gamma_{v}$ with $v \in C \backslash N_{0}$ and $|v|<\varepsilon /\|g\|_{W^{1, \infty}}$.

Proof of Theorem 3.1. The existence of a negligible set $F$ which satisfies the first assertion of the claim follows by Proposition 3.5 of [8]. Up to enlarging this set if necessary, we may as well suppose that $F$ is Borel-measurable.

Set $\widetilde{\varphi}(x, \xi):=\varphi(x, \xi) \chi_{\bar{\Omega} \backslash F}(x)+\beta|\xi| \chi_{F}(x)$ and let $d_{\tilde{\varphi}}$ be the associated distance defined according to (10). Since $\mathbb{L}_{\tilde{\varphi}}(\gamma)=\mathbb{L}_{\varphi}(\gamma)$ if $\gamma$ is transversal to $F$, we obviously have that $d_{\widetilde{\varphi}} \leq \widetilde{d}_{\varphi}$. We want to prove the reverse inequality. It will be enough to show that for every $\gamma \in \operatorname{Lip}_{x, y}$ and every $\varepsilon>0$ there exists a curve $\gamma_{\varepsilon} \in \operatorname{Lip}_{x, y}$ transversal to $F$ such that $\varepsilon+\mathbb{L}_{\tilde{\varphi}}(\gamma)>\mathbb{L}_{\varphi}\left(\gamma_{\varepsilon}\right)$, with $x$ and $y$ arbitrarily chosen in $\bar{\Omega}$. Then, let $\gamma \in \operatorname{Lip}_{x, y}$ and let $A:=\{t \in(0,1) \mid \gamma(t) \in F\}$. Fix $\varepsilon>0$ and assume $0<\mathcal{L}^{1}(A)<1$, being the other cases trivial. Choose an open set $J \supset A$ in $(0,1)$ such that $\mathcal{L}^{1}(J \backslash A)<\varepsilon$. The open set $J$ is a countable disjoint union of intervals of the form $J_{k}:=\left(a_{k}, b_{k}\right)$ with $k \in \mathbb{N}$. Applying Lemma 3.2, we choose, for each $k \in \mathbb{N}$, a curve $\sigma_{k}:\left[a_{k}, b_{k}\right] \rightarrow \bar{\Omega}$ transversal to $F$ such that $\sigma_{k}\left(a_{k}\right)=\gamma\left(a_{k}\right), \sigma_{k}\left(b_{k}\right)=\gamma\left(b_{k}\right)$ and $\left\|\sigma_{k}-\gamma\right\|_{W^{1, \infty}\left(J_{k}, \bar{\Omega}\right)}<\varepsilon / 2^{k}$. For each $n \in \mathbb{N}$ let us set:

$$
\gamma^{n}(t):= \begin{cases}\sigma_{k}(t) & \text { if } t \in\left[a_{k}, b_{k}\right] \text { for each } k \leq n \\ \gamma(t) & \text { otherwise. }\end{cases}
$$

Let $\gamma_{\varepsilon}$ be the curve defined by (19) with $n=+\infty$. It is easily seen that $\left(\gamma^{n}\right)_{n}$ is a Cauchy sequence in $W^{1, \infty}(I, \bar{\Omega})$ and uniformly converges to $\gamma_{\varepsilon}$, which is therefore Lipschitz too. We claim that $\gamma_{\varepsilon}$ is the desired curve. Indeed, it connects $x$ and $y$ in $\bar{\Omega}$ and is transversal to $F$ by construction. Moreover we have:

$$
\begin{aligned}
\int_{J_{k}}\left(\varphi\left(\sigma_{k}, \dot{\sigma}_{k}\right)-\widetilde{\varphi}(\gamma, \dot{\gamma})\right) \mathrm{d} t & \leq \beta\left\|\dot{\sigma}_{k}\right\|_{\infty} \mathcal{L}^{1}\left(J_{k} \backslash A\right)+\int_{J_{k} \cap A} \beta\left(\left|\dot{\sigma}_{k}(t)\right|-|\dot{\gamma}(t)|\right) \mathrm{d} t \\
& <C \mathcal{L}^{1}\left(J_{k} \backslash A\right)+\beta \frac{\varepsilon}{2^{k}}
\end{aligned}
$$

where $C$ is a constant depending only on $\beta$ and $\|\dot{\gamma}\|_{\infty}$. Therefore

$$
\mathbb{L}_{\varphi}\left(\gamma_{\varepsilon}\right)-\mathbb{L}_{\tilde{\varphi}}(\gamma)=\sum_{k=1}^{+\infty} \int_{J_{k}}\left(\varphi\left(\sigma_{k}, \dot{\sigma}_{k}\right)-\widetilde{\varphi}(\gamma, \dot{\gamma})\right) \mathrm{d} t<C \mathcal{L}^{1}(J \backslash A)+\beta \varepsilon<(C+\beta) \varepsilon
$$

and the claim follows.

Remark 3.3. Let us remark that formula (17) is invariant with respect to modifications of the function $\varphi$ on negligible subsets of $\bar{\Omega}$. Therefore, since $\widetilde{\varphi}(x, \xi)=\varphi(x, \xi)$ for $\mathcal{L}^{N}$-a.e. $x \in \bar{\Omega}$ and every $\xi \in \mathbb{R}^{N}$, we also have that $\widetilde{d}_{\widetilde{\varphi}}=\widetilde{d}_{\varphi}=d_{\widetilde{\varphi}}$.

Corollary 3.4. $\widetilde{\mathcal{D}}$ is a proper subset of $\mathcal{D}$.

Proof. Let $\varphi(x, \xi)$ be equal to $\alpha|\xi|$ on a segment $\Gamma$ contained in $\Omega$ and $\beta|\xi|$ elsewhere, and let $d:=d_{\varphi}$ be the distance associated to $\varphi$ through (10). If $d$ belonged to $\widetilde{\mathcal{D}}$, by taking into account Theorem 3.1 and Remark 3.3 , we would have $d=d_{\psi}=\widetilde{d}_{\psi}$ for a function $\psi \in \mathcal{M}$. Proposition 2.9 and the definition of $\varphi_{d}$ would imply $\psi(x, \xi) \geq \varphi_{d}(x, \xi)=\beta|\xi|$ for $\mathcal{L}^{N}$-a.e. $x \in \Omega$ and every $\xi \in \mathbb{R}^{N}$, hence $\psi(x, \xi)=\beta|\xi| \mathcal{L}^{N}$-a.e. on $\bar{\Omega}$. Then, by Remark 3.3, we would have $d=\widetilde{d}_{\psi}=\beta d_{\Omega}$, which is obviously impossible since $d(x, y)=\alpha|x-y|$ if $x$ and $y$ belong to the segment $\Gamma$.

Definitions (10) and (17) individuate two different ways to derive a distance from a given $\varphi \in \mathcal{M}$. In general, we have that $d_{\varphi} \leq \widetilde{d}_{\varphi}$, and the inequality may be strict, as shown by the function $\varphi$ defined in the proof of Corollary 3.4. It seems a difficult task to characterize the functions $\varphi$ for which equality holds. We therefore 
restrict to look for sufficient conditions which entail equivalence between the two definitions. The next two propositions show that the upper semicontinuity property of the length functional $\mathbb{L}_{\varphi}$ plays a role in this issue. These results are essentially known [13-15]; they have been restated here for the reader's convenience.

Proposition 3.5. Let $\varphi \in \mathcal{M}$ be such that the length functional $\mathbb{L}_{\varphi}$ is upper semicontinuous on $W^{1, \infty}(I, \bar{\Omega})$ with respect to the strong topology. Then $d_{\varphi}=\widetilde{d}_{\varphi}$.

Proof. Let $F$ be a Borel negligible subset of $\bar{\Omega}$ satisfying (18), according to Theorem 3.1. Fix $x$ and $y$ in $\bar{\Omega}$ and let $\gamma \in \operatorname{Lip}_{x, y}$. By applying Lemma 3.2, we find a sequence of curves $\left(\gamma_{n}\right)_{n} \subset \operatorname{Lip}_{x, y}$ transversal to $F$ which converges to $\gamma$ in $W^{1, \infty}(I, \bar{\Omega})$. By the upper semicontinuity of $\mathbb{L}_{\varphi}$ we get

$$
\mathbb{L}_{\varphi}(\gamma) \geq \limsup _{n \rightarrow+\infty} \mathbb{L}_{\varphi}\left(\gamma_{n}\right) \geq \widetilde{d}_{\varphi}(x, y)
$$

By taking the infimum over all possible curves in $\operatorname{Lip}_{x, y}$ we obtain $d_{\varphi}(x, y) \geq \widetilde{d}_{\varphi}(x, y)$ and hence the claim.

Proposition 3.6. Let $\varphi \in \mathcal{M}$ be upper semicontinuous in $\bar{\Omega} \times \mathbb{R}^{N}$. Then the length functional $\mathbb{L}_{\varphi}$ is upper semicontinuous on $W^{1, \infty}(I, \bar{\Omega})$ with respect to the strong topology. In particular, $d_{\varphi}=\widetilde{d}_{\varphi}$.

Proof. Let $\left(\gamma_{n}\right)_{n}$ be a sequence in $W^{1, \infty}(I, \bar{\Omega})$ which strongly converges to $\gamma$. Using Fatou's lemma and the upper semicontinuity of $\varphi$ we get

$$
\int_{0}^{1} \varphi(\gamma, \dot{\gamma}) \mathrm{d} t \geq \int_{0}^{1} \limsup _{n \rightarrow+\infty} \varphi\left(\gamma_{n}, \dot{\gamma}_{n}\right) \mathrm{d} t \geq \limsup _{n \rightarrow+\infty} \int_{0}^{1} \varphi\left(\gamma_{n}, \dot{\gamma}_{n}\right) \mathrm{d} t
$$

and so the claim.

In view of the results obtained in [11] and of what seen so far, we can prove what follows.

Proposition 3.7. $\widetilde{\mathcal{D}}$ is a proper and dense subset of $\mathcal{D}$. In particular, it is not closed.

Proof. Proposition 3.6 implies that $\widetilde{\mathcal{D}}$ contains the distances $d_{\varphi}$ with $\varphi \in \mathcal{M}$ continuous, so the density follows by Theorem 4.1 in [11].

In conclusion, the upper semicontinuity of $\varphi$ is a sufficient condition to entail equivalence of (10) and (17). In fact, in the counterexample given in Remark 3.3 the function $\varphi$ we defined was lower semicontinuous. On the other hand, it is clear that the condition we have found is far from being optimal: if the set where $\varphi$ fails to be upper semicontinuous is not too bad, equivalence between (10) and (17) still holds. A naive example of this situation is given by a function $\varphi(x, \xi)$ of the form $a(x)|\xi|$ with $a$ equal to 2 on $\mathbb{R} \times(0,+\infty)$ and to 1 on $\mathbb{R} \times(-\infty, 0]$. The proposition that follows generalizes this idea.

Proposition 3.8. Assume that $\bar{\Omega}:=\cup_{i=1}^{m} \bar{\Omega}_{i}$, where the sets $\Omega_{i}$ are bounded domains with Lipschitz boundaries such that $\bar{\Omega}_{i} \cap \bar{\Omega}_{j}=\partial \Omega_{i} \cap \partial \Omega_{j}$ if $i \neq j$, and every $x \in \Omega$ belongs to at most two subdomains $\bar{\Omega}_{i}$. Let $\varphi \in \mathcal{M}$ and suppose that $\varphi$ is upper semicontinuous in each $\Omega_{i}$. Moreover, let us assume that for every $x \in \cup_{i=1}^{m} \partial \Omega_{i}$ there exist an index $i_{0}$ and a real number $\rho>0$ such that $x \in \partial \Omega_{i_{0}}$ and $\varphi$ is upper semicontinuous in $\bar{\Omega}_{i_{0}} \cap B_{\rho}(x)$. Then $d_{\varphi}(x, y)=\widetilde{d}_{\varphi}(x, y)$ on $\bar{\Omega} \times \bar{\Omega}$.

Proof. First remark that by compactness the number $\rho>0$ in the above assumption can be chosen independent on $x$.

Let $F$ be a Borel negligible subset of $\bar{\Omega}$ satisfying (18) in Theorem 3.1. It will be enough to show that for every $\gamma \in \operatorname{Lip}_{x, y}$ and every $\varepsilon>0$ there exists a curve $\gamma_{\varepsilon} \in \operatorname{Lip}_{x, y}$ transversal to $F$ such that $\mathbb{L}_{\varphi}(\gamma)+\varepsilon>\mathbb{L}_{\varphi}\left(\gamma_{\varepsilon}\right)$, with $x, y \in \bar{\Omega}$.

Let us then take a curve $\gamma \in \operatorname{Lip}_{x, y}$ and fix $\varepsilon>0$. If $\gamma(I)$ is contained in $\Omega_{i}$ for some index $i$, one can apply Lemma 3.2 with $\Omega:=\Omega_{i}$ and conclude by remarking that $\mathbb{L}_{\varphi}$ is upper semicontinuous in $W^{1, \infty}\left(I, \Omega_{i}\right)$. 
Otherwise, there exists a point $x \in \gamma(I) \cap \bigcup_{i=1}^{m} \partial \Omega_{i}$. Up to subdividing $\gamma(I)$ into a finite number of small subarcs, we can assume that $\gamma$ lies in $B_{r}(x) \cap \bar{\Omega}$, where $r<\rho$ is a sufficiently small radius. The case of $x$ belonging to $\partial \Omega_{i}$ for just one index $i$ is easy to deal with: for $r$ small enough $B_{r}(x) \cap \bar{\Omega}=B_{r}(x) \cap \bar{\Omega}_{i}$ for some index $i$ and $\varphi$ is upper semicontinuous in $B_{r}(x) \cap \bar{\Omega}_{i}$ by hypothesis, so $\mathbb{L}_{\varphi}$ is upper semicontinuous in $W^{1, \infty}\left(I, B_{r}(x) \cap \bar{\Omega}\right)$ and the claim follows by applying Lemma 3.2 again.

Let us then suppose that $x$ belongs to $\gamma(I) \cap \partial \Omega_{i}$ for two distinct $i$. Up to reordering the indexes and to choosing a smaller $r$, we may suppose $x \in \partial \Omega_{1} \cap \partial \Omega_{2}, B_{r}(x) \subset \Omega, B_{r}(x) \cap \Omega_{i}=\emptyset$ for each $i \geq 3$ and $\varphi$ upper semicontinuous in $\bar{\Omega}_{1} \cap B_{r}(x)$. Assume also that $r$ has been chosen so small that there exists a cone $C:=\left\{v \in B_{\delta}(0)|\langle v, \xi\rangle>\delta| v \mid\right\}$ (for suitable $\delta>0$ and $\xi \in \mathbb{S}^{N-1}$ ) such that $z+C \subset \Omega_{1}$ for every $z \in \partial \Omega_{1} \cap B_{r}(x)$. Arguing as in the proof of Lemma 3.2, we can take a sequence $\left(v_{n}\right)_{n} \subset C$ converging to 0 such that the curves $\gamma_{n}(t):=\gamma(t)+v_{n} \sin (\pi t)$ are transversal to $F$ and $\left\|\gamma-\gamma_{n}\right\|_{W^{1, \infty}(I, \bar{\Omega})} \leq 2\left|v_{n}\right|$. Let us set $I_{1}:=\left\{t \in I \mid \gamma(t) \in \bar{\Omega}_{1}\right\}$ and $I_{2}:=\left\{t \in I \mid \gamma(t) \in \Omega_{2}\right\}$. Notice that, if $\gamma(t) \in \bar{\Omega}_{1}$, then $\gamma_{n}(t):=\gamma(t)+v_{n} \sin (\pi t) \in \bar{\Omega}_{1}$ for every $n \in \mathbb{N}$, since the translation by the vector $\sin (\pi t) v_{n}$ has the effect of moving points on $\partial \Omega_{1}$ inside $\Omega_{1}$. On the other hand, it is clear that if $\gamma(t) \in \Omega_{2}$ then $\gamma_{n}(t) \in \Omega_{2}$ for $n$ big enough. Therefore, by Fatou's Lemma and taking into account the upper semicontinuity properties enjoyed by $\varphi$, we get

$$
\begin{aligned}
\int_{0}^{1} \varphi(\gamma, \dot{\gamma}) \mathrm{d} t= & \int_{I_{1}} \varphi(\gamma, \dot{\gamma}) \mathrm{d} t+\int_{I_{2}} \varphi(\gamma, \dot{\gamma}) \mathrm{d} t \geq \int_{I_{1}} \limsup _{n \rightarrow+\infty} \varphi\left(\gamma_{n}, \dot{\gamma}_{n}\right) \mathrm{d} t \\
& +\int_{I_{2}} \limsup _{n \rightarrow+\infty} \varphi\left(\gamma_{n}, \dot{\gamma}_{n}\right) \mathrm{d} t \geq \limsup _{n \rightarrow+\infty} \int_{0}^{1} \varphi\left(\gamma_{n}, \dot{\gamma}_{n}\right) \mathrm{d} t .
\end{aligned}
$$

The claim follows by setting $\gamma_{\varepsilon}:=\gamma_{n}$ for $n$ big enough.

\section{Monge SOlutions: DEFinitions AND MAin PROPERTIES}

In this section we study the main properties of Monge sub and supersolutions for the equation

$$
H(x, D u)=0 \quad x \in \Omega \subset \mathbb{R}^{N} .
$$

We will deal with Hamiltonians $H$ satisfying the following set of Assumptions $(\mathrm{H})$ :

(H1) $H: \bar{\Omega} \times \mathbb{R}^{N} \rightarrow \mathbb{R}$ is Borel-measurable;

(H2) for every $x \in \bar{\Omega}$ the 0 -sublevel set

$$
Z(x):=\left\{p \in \mathbb{R}^{N} \mid H(x, p) \leq 0\right\}
$$

is closed and convex. Moreover $\partial Z(x)=\left\{p \in \mathbb{R}^{N} \mid H(x, p)=0\right\}$ for all $x \in \bar{\Omega}$;

(H3) there exist $\alpha, \beta>0$ such that $B_{\alpha}(0) \subset Z(x) \subset B_{\beta}(0)$ for every $x \in \bar{\Omega}$.

We recall the definition of optical length function relative to the Hamiltonian $H$, that is the map $S: \bar{\Omega} \times \bar{\Omega} \rightarrow \mathbb{R}$ defined by:

$$
S(x, y):=\inf \left\{\int_{0}^{1} \sigma(\gamma(t), \dot{\gamma}(t)) \mathrm{d} t \mid \gamma \in \operatorname{Lip}_{x, y}\right\}
$$

for every $x, y \in \bar{\Omega}$, where $\sigma$ is the support function of the 0 -sublevel set $Z(x)$, namely

$$
\sigma(x, \xi):=\sup \{\langle-\xi, p\rangle \mid p \in Z(x)\} .
$$

Note that, when it will be needed, given an Hamiltonian $H$, we will respectively denote by $Z_{H}(x), S_{H}(x, y)$, $\sigma_{H}(x, \xi)$ the corresponding 0-sublevel set (21), optical length function (22) and support function (23). The definition of Monge solution is given as follows. 
Definition 4.1. Let $u \in C(\Omega)$. We say that $u$ is a Monge solution (resp. subsolution, supersolution) of (20) in $\Omega$, if for each $x_{0} \in \Omega$ there holds

$$
\liminf _{x \rightarrow x_{0}} \frac{u(x)-u\left(x_{0}\right)+S\left(x_{0}, x\right)}{\left|x-x_{0}\right|}=0 \quad(\text { resp. } \geq, \leq) .
$$

The general results obtained in Section 2 will be now specialized to derive the main properties of the optical length function $S$ defined in (22). Note that $S$ is indeed the non-symmetric distance $d_{\varphi}$ defined in (10) with $\varphi:=\sigma$. We start by studying the regularity of $\sigma$ in the following lemma.

Lemma 4.2. If $H$ is an Hamiltonian satisfying $(\mathrm{H})$, then the function $\sigma: \bar{\Omega} \times \mathbb{R}^{N} \rightarrow \mathbb{R}_{+}$belongs to $\mathcal{M}$ and $\sigma(x, \cdot)$ is convex on $\mathbb{R}^{N}$ for every $x \in \bar{\Omega}$.

Moreover

(i) if $H(\cdot, p)$ is upper semicontinuous on $\bar{\Omega}$ for every $p \in \mathbb{R}^{N}$, then $\sigma(\cdot, \xi)$ is lower semicontinuous on $\bar{\Omega}$ for every $\xi \in \mathbb{R}^{N}$

(ii) if $H(\cdot, p)$ is lower semicontinuous on $\bar{\Omega}$ for every $p \in \mathbb{R}^{N}$, then $\sigma(\cdot, \xi)$ is upper semicontinuous on $\bar{\Omega}$, for every $\xi \in \mathbb{R}^{N}$.

Proof. In order to prove that $\sigma \in \mathcal{M}$, it will be enough to show $\sigma$ is Borel measurable, since all the other properties immediately follow from the definition of $\sigma$ and Assumptions $(\mathrm{H})$. Let $\left(p_{i}\right)_{i}$ be a countable dense subset of $\mathbb{R}^{N}$. By $(\mathrm{H} 2)$ and (H3), it is easily seen that

$$
\sigma(x, \xi)=\sup _{i \in \mathbb{N}}\left\{\left\langle-\xi, p_{i}\right\rangle \mid p_{i} \in Z(x)\right\}=\sup _{i \in \mathbb{N}}\left\{\left\langle-\xi, p_{i}\right\rangle \chi_{E_{i}}(x)\right\}
$$

where $E_{i}:=\left\{x \in \bar{\Omega} \mid H\left(x, p_{i}\right)<0\right\}$. Notice that, by Assumption (H1), $E_{i}$ is a Borel set, hence each function $(x, \xi) \mapsto\left\langle-\xi, p_{i}\right\rangle \chi_{E_{i}}(x)$ is Borel-measurable and the claim follows. In order to prove (i), we remark that, by Assumption (H3), one can replace the functions $\left\langle-\xi, p_{i}\right\rangle \chi_{E_{i}}(x)$ with $\left(\left\langle-\xi, p_{i}\right\rangle \vee \alpha|\xi|\right) \chi_{E_{i}}(x)$ in $(25)$ without affecting the equality. Then, as $E_{i}$ is open for every $i \in \mathbb{N}$, each function $x \mapsto\left(\left\langle-\xi, p_{i}\right\rangle \vee \alpha|\xi|\right) \chi_{E_{i}}(x)$ is lower semicontinuous for every fixed $\xi \in \mathbb{R}^{N}$, and so is $\sigma(\cdot, \xi)$. The remainder of the claim easily follows by Assumptions $(\mathrm{H})$ and the definition of support function $\sigma$.

Remark 4.3. Comparing the above lemma with Proposition 2.2, we obtain that the function $S$ is well-defined. Moreover (see also Rem. 2.3), it is a non-symmetric geodesic distance such that:

(i) $\alpha|x-y| \leq S(x, y)$ for every $x, y \in \bar{\Omega}$;

(ii) $S(x, y) \leq \beta|x-y|$ locally in $\Omega$;

(iii) $S$ is Lipschitz on $\bar{\Omega} \times \bar{\Omega}$, with Lipschitz constant equal to $2 \beta C$, where $C \geq 1$ is the Lipschitz constant of $\partial \Omega$.

In particular, by Proposition 2.1, for every $x, y \in \bar{\Omega}$, there exists a curve $\gamma \in \operatorname{Lip}_{x, y}$ such that $S(x, y)=\mathrm{L}_{S}(\gamma)$, where $\mathrm{L}_{S}(\gamma)$ is the length of the curve $\gamma$ defined according to (8) for the non-symmetric distance $S$.

We want to show now that the definitions of Monge sub and supersolution are consistent with those given in the viscosity sense in the classical setting of a continuous Hamiltonian.

Definition 4.4. A function $u \in C(\Omega)$ is a viscosity subsolution of (20) in $\Omega$ if

$$
H\left(x_{0}, q\right) \leq 0 \quad \text { for every } x_{0} \in \Omega \text { and every } q \in D^{+} u\left(x_{0}\right)
$$

Similarly, $u \in C(\Omega)$ is a viscosity supersolution of (20) in $\Omega$ if

$$
H\left(x_{0}, q\right) \geq 0 \quad \text { for every } x_{0} \in \Omega \text { and every } q \in D^{-} u\left(x_{0}\right)
$$


We say that $u \in C(\Omega)$ is a viscosity solution of (20) in $\Omega$ if it is both a subsolution and a supersolution in the viscosity sense. Here we have denoted by $D^{+} u\left(x_{0}\right)$ and $D^{-} u\left(x_{0}\right)$ the classical superdifferential and subdifferential of $u$ at $x_{0}$.

Proposition 4.5. Let $H$ be a continuous Hamiltonian satisfying $(\mathrm{H})$. Then $v \in C(\Omega)$ is a Monge supersolution (resp. subsolution) of (20) if and only if it is a viscosity supersolution (resp. subsolution) of (20).

Proof. To prove that any viscosity supersolution (resp. subsolution) in $C(\Omega)$ is a Monge supersolution (resp. subsolution), one can argue as in [19].

Conversely, let $v \in C(\Omega)$ be a Monge supersolution. Let $x_{0} \in \Omega$ and $q \in D^{-} v\left(x_{0}\right)$. By definition we have

$$
0 \geq \liminf _{x \rightarrow x_{0}} \frac{v(x)-v\left(x_{0}\right)+S\left(x_{0}, x\right)}{\left|x-x_{0}\right|} \geq \liminf _{x \rightarrow x_{0}}\left(\left\langle q, \frac{x-x_{0}}{\left|x-x_{0}\right|}\right\rangle+\frac{S\left(x_{0}, x\right)}{\left|x-x_{0}\right|}\right) .
$$

Let $\left(x_{n}\right)_{n}$ be a minimizing sequence for the most right-hand side of (26). We set

$$
\xi_{n}:=\frac{x_{n}-x_{0}}{\left|x_{n}-x_{0}\right|}, \quad t_{n}:=\left|x_{n}-x_{0}\right| .
$$

Up to subsequences, we have that $\xi_{n} \rightarrow \xi \in \mathbb{S}^{N-1}$. Moreover

$$
\liminf _{n \rightarrow+\infty} \frac{S\left(x_{0}, x_{0}+t_{n} \xi_{n}\right)}{t_{n}}=\liminf _{n \rightarrow+\infty} \frac{S\left(x_{0}, x_{0}+t_{n} \xi\right)}{t_{n}} \geq \sigma\left(x_{0}, \xi\right) .
$$

Indeed, the first equality comes from

$$
\left|\frac{S\left(x_{0}, x_{0}+t_{n} \xi_{n}\right)-S\left(x_{0}, x_{0}+t_{n} \xi\right)}{t_{n}}\right| \leq \beta\left|\xi_{n}-\xi\right|,
$$

while the second follows by the continuity of $H$ (and therefore of $\sigma$ by Lem. 4.2) and Proposition 2.9(i). Therefore by (26) we obtain

$$
0 \geq \lim _{n \rightarrow+\infty}\left(\left\langle q, \xi_{n}\right\rangle+\frac{S\left(x_{0}, x_{0}+t_{n} \xi_{n}\right)}{t_{n}}\right) \geq\langle q, \xi\rangle+\sigma\left(x_{0}, \xi\right),
$$

that is $\langle-\xi, q\rangle \geq \sigma\left(x_{0}, \xi\right)=\sup \left\{\langle-\xi, p\rangle \mid p \in Z\left(x_{0}\right)\right\}$. In view of Assumptions (H2), (H3) that easily implies $H\left(x_{0}, q\right) \geq 0$.

Let $v \in C(\Omega)$ be a Monge subsolution. Let $x_{0} \in \Omega$ and $q \in D^{+} v\left(x_{0}\right)$. We have

$$
0 \leq \liminf _{x \rightarrow x_{0}} \frac{v(x)-v\left(x_{0}\right)+S\left(x_{0}, x\right)}{\left|x-x_{0}\right|} \leq \limsup _{x \rightarrow x_{0}}\left(\left\langle q, \frac{x-x_{0}}{\left|x-x_{0}\right|}\right\rangle+\frac{S\left(x_{0}, x\right)}{\left|x-x_{0}\right|}\right) .
$$

If it were $H\left(x_{0}, q\right)>0$, by Hahn-Banach theorem there would exist a vector $\xi \in \mathbb{S}^{N-1}$ such that $\langle-\xi, q\rangle>$ $\sup \left\{\langle-\xi, p\rangle \mid p \in Z\left(x_{0}\right)\right\}=\sigma\left(x_{0}, \xi\right)$. But that is impossible, since, by taking the sequence $x_{n}=x_{0}+t_{n} \xi$ with $t_{n}=1 / n$, from inequality (28) and Proposition 2.9(ii) we get

$$
0 \leq\langle q, \xi\rangle+\limsup _{n \rightarrow+\infty} \frac{S\left(x_{0}, x_{0}+t_{n} \xi\right)}{t_{n}} \leq\langle q, \xi\rangle+\sigma\left(x_{0}, \xi\right) .
$$

In the measurable setting, the following pointwise description of the behavior of Monge sub and supersolutions holds.

Proposition 4.6. Let $v$ be a Lipschitz function in $\Omega$ and $H$ satisfy $(\mathrm{H})$. 
(i) If $v$ is a Monge subsolution of (20), then it is a Lipschitz subsolution, i.e.

$$
H(x, D v(x)) \leq 0 \quad \text { for } \mathcal{L}^{N} \text {-a.e. } x \in \Omega .
$$

(ii) If $\sigma(\cdot, \xi)$ is lower semicontinuous for every $\xi \in \mathbb{R}^{N}$ and $v$ is a Monge supersolution of (20), then it is a Lipschitz supersolution, i.e.

$$
H(x, D v(x)) \geq 0 \quad \text { for } \mathcal{L}^{N} \text {-a.e. } x \in \Omega .
$$

In particular, a Monge solution is a Lipschitz solution, i.e. it solves (20) almost everywhere in $\Omega$.

For the proof, the reader may follow word by word that of Proposition 4.5, using Proposition 2.9(i) instead of the continuity of the support function $\sigma$.

The next proposition says that any Monge subsolution is locally 1-Lipschitz continuous with respect to the non-symmetric distance $S$ (cf. [19], Lem. 3.1).

Proposition 4.7. Let $H$ be an Hamiltonian satisfying $(\mathrm{H})$ and $u \in C(\Omega)$ be a Monge subsolution of (20). Then $u$ is Lipschitz in $\Omega$ and $|D u| \leq \beta$ a.e. in $\Omega$. Moreover, for every $x_{0} \in \Omega$ there exists an $r>0$, depending only on $\operatorname{dist}\left(x_{0}, \partial \Omega\right), \alpha, \beta$, such that

$$
u(x)-u(y) \leq S(x, y) \quad \text { for every } x, y \in B_{r}\left(x_{0}\right)
$$

Proof. First remark that the function $u$ is Lipschitz continuous on $\Omega$ with $|D u| \leq \beta$ a.e. in $\Omega$. Indeed, by the fact that $u$ is a Monge subsolution and Remark 4.3, we have that $u$ is a Monge subsolution of $|D v|=\beta$, hence a (classical) viscosity subsolution. This remark, together with the Lipschitz character of $\partial \Omega$, proves the assertion.

Now, fix a point $x_{0} \in \Omega$. We can choose an $r>0$ small enough so that every optimal path for $S(x, y)$ with $x, y \in B_{r}\left(x_{0}\right)$ lies inside $\Omega$. Observe that $r$ is only dependent on $\operatorname{dist}\left(x_{0}, \partial \Omega\right), \alpha, \beta$ (cf. Rem. 4.3). Fix $x, y \in B_{r}\left(x_{0}\right)$ and take an optimal path $\gamma \in \operatorname{Lip}_{x, y}$ for $S(x, y)$. By Remark 4.3 the function $f(t):=S(x, \gamma(t))$ is Lipschitz continuous. Therefore the function $u_{\circ} \gamma(t)+f(t)$ is Lipschitz continuous and we can compute its derivative for $\mathcal{L}^{1}$-a.e. $t \in I$. We have then

$$
\begin{aligned}
\frac{\mathrm{d}}{\mathrm{d} t}(u \circ \gamma+f)(t) & =\lim _{s \rightarrow t^{+}} \frac{u(\gamma(s))-u(\gamma(t))+S(x, \gamma(s))-S(x, \gamma(t))}{s-t} \\
& =|\dot{\gamma}(t)| \lim _{s \rightarrow t^{+}} \frac{u(\gamma(s))-u(\gamma(t))+S(\gamma(t), \gamma(s))}{|\gamma(s)-\gamma(t)|} \geq 0
\end{aligned}
$$

for $\mathcal{L}^{1}$-a.e. $t \in I$, where we have used the optimality of $\gamma$ and the definition of Monge subsolution. By integrating the above inequality we get (30), that is the claim.

\section{The COMParison Result And solvability of the Dirichlet Problem}

Our comparison result is stated as follows.

Theorem 5.1 (comparison theorem). Let $H$ be an Hamiltonian satisfying $(\mathrm{H})$ and let $u, v \in C(\bar{\Omega})$ be, respectively, a Monge subsolution and a Monge supersolution of (20) in $\Omega$. If $u \leq v$ on $\partial \Omega$ then $u \leq v$ in $\Omega$.

Proof. By contradiction, assume that the assertion is false. Then the function $\varepsilon u-v$ attains its maximum on $\bar{\Omega}$ at some point $x_{0} \in \Omega$, for $\varepsilon \in(0,1)$ close to 1 . Therefore

$$
\liminf _{x \rightarrow x_{0}} \frac{v(x)-v\left(x_{0}\right)+S\left(x_{0}, x\right)}{\left|x-x_{0}\right|} \geq \liminf _{x \rightarrow x_{0}} \frac{\varepsilon u(x)-\varepsilon u\left(x_{0}\right)+\varepsilon S\left(x_{0}, x\right)+(1-\varepsilon) \alpha\left|x-x_{0}\right|}{\left|x-x_{0}\right|}>0,
$$

in contradiction with $v$ being a Monge supersolution. 
We address now our attention to the Dirichlet problem

$$
\begin{cases}H(x, D u)=0 & \text { in } \Omega \\ u=g & \text { on } \partial \Omega .\end{cases}
$$

More precisely, we will prove that the function $u$ given by the Lax formula

$$
u(x):=\inf _{y \in \partial \Omega}\{S(x, y)+g(y)\} \quad \text { for } x \in \bar{\Omega},
$$

is a Monge solution of the Dirichlet problem (31) according to the following definition.

Definition 5.2. We will say that a function $u \in C(\bar{\Omega})$ is a Monge solution of the Dirichlet problem (31) if it is a Monge solution of equation $H(x, D u)=0$ in $\Omega$ and $u(x)=g(x)$ for each $x \in \partial \Omega$.

Our result is the following.

Theorem 5.3 (solvability of the Dirichlet problem). Let $H$ be an Hamiltonian satisfying $(\mathrm{H})$ and assume that the boundary datum $g: \partial \Omega \rightarrow \mathbb{R}$ satisfies the compatibility condition

$$
g(x)-g(y) \leq S(x, y) \quad \text { for every } x, y \in \partial \Omega .
$$

The function $u$ given by the Lax formula (32) is the unique Monge solution of the Dirichlet problem (31). Moreover, $u$ is the maximal element of the set

$$
\mathcal{S}_{M}:=\{v \in C(\bar{\Omega}) \mid v \text { Monge subsolution of }(20) \text { in } \Omega, v \leq g \text { on } \partial \Omega\}
$$

The effect of the compatibility Condition (33) is that of guaranteeing that the function $u$ defined by (32) attains the boundary datum $g$ on $\partial \Omega$, while the other properties enjoyed by $u$ are actually independent of (33). This fact is underlined by the following

Proposition 5.4. Let $H$ be an Hamiltonian satisfying $(\mathrm{H})$ and $g: \partial \Omega \rightarrow \mathbb{R}$ be a function bounded from below. The function $u$ defined by (32) is Lipschitz continuous on $\bar{\Omega}$. Moreover, $u$ is a Monge solution of (20) in $\Omega$.

Proof. As $g$ is bounded from below, $u$ is well defined on $\bar{\Omega}$ by formula (32). One can check that, by definition, $|u(x)-u(y)| \leq \max \{S(x, y), S(y, x)\}$ on $\bar{\Omega} \times \bar{\Omega}$, therefore $u$ is Lipschitz continuous on $\bar{\Omega}$ (cf. Rem. 4.3), in particular it is of class $C(\bar{\Omega})$.

To show that $u$ is a Monge subsolution, fix $x_{0} \in \Omega$ and an arbitrary sequence $\left(x_{n}\right)_{n}$ in $\Omega$ which converges to $x_{0}$. For every $n \in \mathbb{N}$ choose a point $y_{n} \in \partial \Omega$ such that $u\left(x_{n}\right) \geq S\left(x_{n}, y_{n}\right)+g\left(y_{n}\right)-o\left(\left|x_{0}-x_{n}\right|\right)$. Then

$$
u\left(x_{n}\right)+S\left(x_{0}, x_{n}\right) \geq S\left(x_{0}, y_{n}\right)+g\left(y_{n}\right)-o\left(\left|x-x_{n}\right|\right) \geq u\left(x_{0}\right)-o\left(\left|x-x_{n}\right|\right)
$$

and, by taking the liminf as $n$ goes to $+\infty$ in the above expression, we conclude that $u$ is a Monge subsolution of (20) by the arbitrariness of $\left(x_{n}\right)_{n}$.

Let us prove that $u$ is a Monge supersolution. Fix $x_{0} \in \Omega$ and, for $n \in \mathbb{N}$ big enough, consider the ball $B_{1 / n}\left(x_{0}\right) \subset \Omega$. Choose an $y_{n} \in \partial \Omega$ such that $u\left(x_{0}\right) \geq S\left(x_{0}, y_{n}\right)+g\left(y_{n}\right)-1 / n^{2}$. Let $\gamma_{n} \in \operatorname{Lip}_{x_{0}, y_{n}}$ be an optimal path for $S\left(x_{0}, y_{n}\right)$ and take a point $z_{n} \in \gamma_{n}(I) \cap \partial B_{1 / n}\left(x_{0}\right)$. By definition we have that $u\left(z_{n}\right) \leq S\left(z_{n}, y_{n}\right)+g\left(y_{n}\right)$. Hence, using also the optimality of $\gamma_{n}$, we have

$$
u\left(z_{n}\right)-u\left(x_{0}\right) \leq S\left(z_{n}, y_{n}\right)-S\left(x_{0}, y_{n}\right)+1 / n^{2}=-S\left(x_{0}, z_{n}\right)+1 / n^{2} .
$$

This implies

$$
\liminf _{n \rightarrow+\infty} \frac{u\left(z_{n}\right)-u\left(x_{0}\right)+S\left(x_{0}, z_{n}\right)}{\left|z_{n}-x_{0}\right|} \leq \liminf _{n \rightarrow+\infty} \frac{1}{n}=0
$$

which obviously implies that $u$ is a Monge supersolution. 
Proof of Theorem 5.3. Uniqueness in the class $C(\bar{\Omega})$ is a consequence of the comparison theorem. By Proposition 5.4 we have that the function $u$ defined by (32) is Lipschitz continuous on $\bar{\Omega}$, in particular of class $C(\bar{\Omega})$, and is a Monge solution of (20) in $\Omega$. We have, by definition, that $u(x) \leq g(x)$ for every $x \in \partial \Omega$ (just choose $y=x$ in formula (32)), while the opposite inequality holds by the compatibility Condition (33). Hence $u=g$ on $\partial \Omega$, therefore $u$ is the unique solution of class $C(\bar{\Omega})$ of the Dirichlet problem (31). Last, the maximality of $u$ in the set $\mathcal{S}_{M}$ trivially follows by the Comparison Theorem.

\section{The StABILITy Result}

We start this section by introducing a suitable convergence on Hamiltonians under which we will prove a stability result for Monge solutions.

Definition 6.1. Let $\left(H_{n}\right)_{n}, H$ be Hamiltonians satisfying Assumptions $(\mathrm{H})$ and $\left(S_{n}\right)_{n}$ and $S$ be the relative optical length functions defined according to (22). We say that $H_{n} \tau$-converges to $H$ and write $H_{n} \stackrel{\tau}{\longrightarrow} H$ if $\left(S_{n}\right)_{n}$ converges uniformly to $S$ on $\bar{\Omega} \times \bar{\Omega}$.

Remark 6.2. Note that the convergence of the Hamiltonians above defined is equivalent, by Theorem 3.1 in [6], to the $\Gamma$-convergence of the length functionals $\left(\mathrm{L}_{S_{n}}\right)_{n}$ to the length functional $\mathrm{L}_{S}$ with respect to the uniform convergence of paths. This, in fact, mainly motivates our definition.

Since our definition does not give a condition one can check on the sequence $\left(H_{n}\right)_{n}$, we will see, in the next proposition, which conditions on the Hamiltonians imply $H_{n} \stackrel{\tau}{\longrightarrow} H$.

Proposition 6.3. Let the Hamiltonians $H,\left(H_{n}\right)_{n}$ satisfy $(\mathrm{H})$. Then $H_{n} \stackrel{\tau}{\longrightarrow} H$ if one of the following conditions holds:

(i) $\left(H_{n}\right)_{n}$ converges uniformly to $H$ on $\bar{\Omega} \times B_{\beta}(0)$.

(ii) For each $n \in \mathbb{N}$ and $p \in B_{\beta}(0)$ the function $H_{n}(\cdot, p)$ is upper semicontinuous on $\bar{\Omega}$ and $\left(H_{n}\right)_{n}$ converge decreasingly to $H$ on $\bar{\Omega} \times B_{\beta}(0)$.

(iii) $\left(H_{n}\right)_{n}$ converges increasingly to $H$ on $\bar{\Omega} \times B_{\beta}(0)$.

Proof. By Definition 6.1 the claim will be proved if we show that $\left(S_{n}\right)_{n}$ uniformly converges to $S$ in $\bar{\Omega} \times \bar{\Omega}$. This easily follows by applying Proposition 2.7 with $\varphi:=\sigma$ and $\varphi_{n}:=\sigma_{n}$ for each $n \in \mathbb{N}$. Indeed Hypothesis (i), (ii), and (iii) implies (i), (ii), and (iii) respectively in Proposition 2.7 (to obtain (ii) we also use Lem. 4.2), and then we can conclude that the distances associated to $\sigma_{n}$, i.e. $S_{n}$, converge uniformly to the distance associated to $\sigma$, i.e. $S$.

We are now ready to show our stability result.

Theorem 6.4 (stability theorem). Let the Hamiltonians $H,\left(H_{n}\right)_{n}$ satisfy the same set of Assumptions $(\mathrm{H})$ for two fixed positive constants $\alpha, \beta$ (independent of $n \in \mathbb{N}$ ). Suppose that:

1. $H_{n} \stackrel{\tau}{\longrightarrow} H$ as $n \rightarrow \infty$;

2. $u_{n} \in C(\Omega)$ is a Monge solution of $H_{n}\left(x, D u_{n}\right)=0$ in $\Omega$ for each $n \in \mathbb{N}$;

3. the sequence $\left(u_{n}\right)_{n}$ converges uniformly to $u \in C(\Omega)$ on compact subsets of $\Omega$.

Then $u$ is a Monge solution of $H(x, D u)=0$ in $\Omega$.

Proof. Fix a point $x_{0} \in \Omega$. By Proposition 4.7, there exists an $r>0$ independent of $n$ such that (30) holds for each $S_{n}$. Therefore we have

$$
u_{n}(x)=\inf _{y \in \partial B_{r}\left(x_{0}\right)}\left\{S_{n}(x, y)+u_{n}(y)\right\} \quad \text { for every } x \in B_{r}\left(x_{0}\right) .
$$

By Definition $6.1\left(S_{n}\right)_{n}$ converge uniformly to $S$ on $\bar{\Omega} \times \bar{\Omega}$ and, by Hypothesis 3 , $u_{n}$ converge uniformly to $u$ in $B_{r}\left(x_{0}\right)$, thus, letting $n \rightarrow \infty$ in (35) we obtain

$$
u(x)=\inf _{y \in \partial B_{r}\left(x_{0}\right)}\{S(x, y)+u(y)\} \quad \text { for every } x \in B_{r}\left(x_{0}\right) .
$$


So, by Theorem 5.3, $u$ is a Monge solution of $H(x, D u)=0$ in $B_{r}\left(x_{0}\right)$. The claim then follows since $(24)$ is a local property and $x_{0} \in \Omega$ was arbitrary.

We end this section describing an example already studied in [8], Example 7.2. We observe that, with our definitions, a stability result holds, while this is not obtained in [8], as stressed by the authors. Note that the difference is in the definition of the optical length function: indeed, we both consider the same discontinuous Hamiltonian $H$ which is the pointwise limit of a sequence of continuous ones $\left(H_{n}\right)_{n}$, but while, using our definition, the corresponding optical length functions $S_{n}$ converge uniformly to the optical length function $S$ corresponding to $H$, with their definition ( $c f$. also Sect. 7 ) the sequence $\left(L_{n}^{\Omega}\right)_{n}$ do not converge to $L^{\Omega}$ (notice that $S_{n}=L_{n}^{\Omega}$ for each $n \in \mathbb{N}$ as $H_{n}$ are continuous, $c f$. Th. 7.3).

Example 6.5. Let $\Omega:=(0,1) \times(-2,2)$ and consider a sequence of continuous functions $a_{n}: \bar{\Omega} \rightarrow \mathbb{R}$ defined by

$$
a_{n}\left(x_{1}, x_{2}\right):= \begin{cases}1 & \text { if }\left|x_{2}\right| \geq 1 / n \\ 1 / 2+\left|x_{2}\right| n / 2 & \text { otherwise }\end{cases}
$$

The functions $a_{n}$ converge increasingly to the function $a(x):=\chi_{\bar{\Omega}}(x)-1 / 2 \chi_{\Gamma}(x)$ pointwise on $\bar{\Omega} \times \mathbb{R}^{N}$, where $\Gamma$ is the $x_{1}$-axis $\mathbb{R} \times\{0\}$. Let us define the Hamiltonians $H_{n}(x, p):=|p|-a_{n}(x)$ and $H(x, p):=|p|-a(x)$. Obviously, $\left(H_{n}\right)_{n}$ and $H$ satisfy Assumptions $(\mathrm{H})$ with, for instance, $\alpha:=1 / 2$ and $\beta:=1$. By Proposition 6.3(ii), we immediately have that $H_{n} \stackrel{\tau}{\longrightarrow} H$, therefore the Stability Theorem holds. In particular, if $g$ is a continuous function on $\partial \Omega$ satisfying the compatibility Condition (33) for $H$ and $H_{n}$ for each $n \in \mathbb{N}$ (take, for instance $g(x):=1 / 2|x|$ for $x \in \partial \Omega)$, then the Monge solutions $u_{n}$ of the Dirichlet problems

$$
\begin{cases}|D v|=a_{n}(x) & \text { in } \Omega \\ v=g & \text { on } \partial \Omega\end{cases}
$$

are classical viscosity solutions (as the Hamiltonians $H_{n}$ are continuous) and converge uniformly on $\bar{\Omega} \times \bar{\Omega}$ to a function $u$ which is the unique Monge solution of

$$
\begin{cases}|D v|=a(x) & \text { in } \Omega \\ v=g & \text { on } \partial \Omega .\end{cases}
$$

\section{Pointwise Behavior of Monge subsolutions}

In this section we will study the pointwise properties enjoyed by the Monge subsolutions of problem (31) and the relation between Monge and Lipschitz subsolutions, in particular we are interested in investigating maximality properties of the function $u$ defined by the Lax formula (32).

We recall that a function $v: \bar{\Omega} \rightarrow \mathbb{R}$ is said to be a Lipschitz subsolution of the Dirichlet problem (31) if $v \in W^{1, \infty}(\Omega), H(x, D v(x)) \leq 0$ for $\mathcal{L}^{N}$-a.e. $x \in \Omega$ and $v \leq g$ on $\partial \Omega$. It is well known that in the classical context of a continuous Hamiltonian $H$ the function $u$ defined in (32) is the maximum element of the set

$$
\mathcal{S}_{P}:=\left\{v \in W^{1, \infty}(\Omega) \mid H(x, D v(x)) \leq 0 \mathcal{L}^{N} \text {-a.e. } x \in \Omega, v \leq g \text { on } \partial \Omega\right\}
$$

of Lipschitz subsolutions of (31). We wonder if this maximality property is maintained when the Hamiltonian $H$ satisfies the more general hypotheses $(\mathrm{H})$. Indeed, by Proposition 5.4, the function $u$ is a Lipschitz continuous Monge solution of (20), therefore is a Lipschitz subsolution of (31), by Proposition 4.6. But in general it is not the maximum element of $\mathcal{S}_{P}$, not even in the case of a boundary datum $g$ satisfying the compatibility Condition (33), as the following example shows.

Example 7.1. Let $\Omega:=(0,1) \times(-1,1)$ and let $H(x, p):=|p|-a(x)$, where $a(x):=2 \chi_{\bar{\Omega}}(x)-\chi_{\Gamma}(x)$ and $\Gamma$ denotes the $x_{1}$-axis $\mathbb{R} \times\{0\}$. Let $v\left(x_{1}, x_{2}\right):=1 / 2\left|x_{2}\right|+3 / 2\left|x_{1}\right|$. Then the inequality $H(x, D v)<0$ holds true for every differentiability point of $v$ in $\Omega$. Let $u$ be the function given by formula (32) with $g:=v_{\mid \partial \Omega}$. Observe that $g$ satisfy 
the compatibility Condition (33). Nevertheless, we have $u\left(x_{1}, 0\right)=S\left(\left(x_{1}, 0\right),(0,0)\right)=\left|x_{1}\right|<3 / 2\left|x_{1}\right|=v\left(x_{1}, 0\right)$. Hence, $u$ is not the maximum element of $\mathcal{S}_{P}$.

Therefore we are led to seek for sufficient conditions which guarantee the maximality of the function $u$ among all Lipschitz subsolution of (31).

Let $H$ be an Hamiltonian fulfilling Assumptions (H). Following the approach of Camilli and Siconolfi in [8], we define a slightly different optical length function:

$$
L^{\Omega}(x, y):=\sup _{\mathcal{L}^{N}(E)=0}\left\{\inf \left\{\int_{0}^{1} \sigma(\gamma(t), \dot{\gamma}(t)) \mathrm{d} t \mid \gamma \in \operatorname{Lip}_{x, y}, \gamma \text { transversal to } E\right\}\right\}
$$

for every $x, y \in \bar{\Omega}$. We remark that $L^{\Omega}$ is nothing else that the distance $\widetilde{d}_{\sigma}$ defined according to (17). The following result holds [8].

Theorem 7.2. Let $H$ be an Hamiltonian satisfying $(\mathrm{H})$. Assume that $g: \partial \Omega \rightarrow \mathbb{R}$ is a function bounded from below and that $S(x, y)=L^{\Omega}(x, y)$ for every $x, y \in \bar{\Omega}$. Then any Lipschitz subsolution of (31) is a Monge subsolution. Moreover, the function u defined by Lax formula (32) is maximal in $\mathcal{S}_{P}$.

The previous theorem gives a first answer to the question raised before. Unfortunately, the above condition, stated in terms of equality of the optical length functions $S$ and $L^{\Omega}$, is quite indirect. In order to derive conditions on the Hamiltonian, we now use the results obtained in Section 3. The next theorem will indeed follow quite easily from Proposition 3.8. We remark that our result is more general than those obtained by Newcomb and $\mathrm{Su}$ [19], Theorem 5.4 and by Soravia [20], Theorem 4.7: indeed, the Hamiltonian $H$ is not assumed to be piecewise constant in the $x$-variable near the interface of two contiguous subdomains.

Theorem 7.3. Assume that $\bar{\Omega}:=\cup_{i=1}^{m} \bar{\Omega}_{i}$, where the sets $\Omega_{i}$ are bounded domains with Lipschitz boundaries such that $\bar{\Omega}_{i} \cap \bar{\Omega}_{j}=\partial \Omega_{i} \cap \partial \Omega_{j}$ if $i \neq j$, and every $x \in \Omega$ belongs to at most two subdomains $\bar{\Omega}_{i}$.

Let $H$ be an Hamiltonian satisfying $(\mathrm{H})$ and lower semicontinuous in $\Omega_{i} \times \mathbb{R}^{N}$ for each $i$. Moreover, assume that for every $x \in \cup_{i=1}^{m} \partial \Omega_{i}$ there exist an index $i_{0}$ and a real number $\rho>0$ such that $x \in \partial \Omega_{i_{0}}$ and $H$ is lower semicontinuous in $\bar{\Omega}_{i_{0}} \cap B_{\rho}(x)$.

Then $S(x, y)=L^{\Omega}(x, y)$ for every $x, y \in \bar{\Omega}$. In particular, the claim of Theorem 7.2 holds.

Proof. The claim directly follows by applying Proposition 3.8 with $\varphi:=\sigma\left(\right.$ as $S=d_{\sigma}$ and $\left.L^{\Omega}=\widetilde{d}_{\sigma}\right)$. Since the hypotheses on $\Omega$ are the same, we only have to check those on $\sigma$. Since $\sigma(x, \cdot)$ is convex on $\mathbb{R}^{N}$ for every $x \in \bar{\Omega}$, when checking the upper semicontinuity properties of $\sigma$, we can reduce to consider the function $\sigma(\cdot, \xi)$ for every fixed $\xi \in \mathbb{R}^{N}$. Now, it is easy to prove that $\sigma(\cdot, \xi)$ is upper semicontinuous on $X$ if $H$ is lower semicontinuous on $X \times \mathbb{R}^{N}$, being $X$ a subspace of $\mathbb{R}^{N}$ and $\xi$ a fixed vector in $\mathbb{R}^{N}$. This argument, applied with $X:=\Omega_{i}$ and $X:=\bar{\Omega}_{i_{0}} \cap B_{\rho}(x)$ with $x, i_{o}$ and $\rho$ as in the statement of the theorem, shows that the assumptions of Proposition 3.8 are fulfilled.

Another question that could be raised is whether the last part of the claim of Theorem 5.3 is still true even when $g$ does not satisfy the compatibility Condition (33), that is we wonder if the function $u$ defined by (32) is the maximum element of the set $\mathcal{S}_{M}$ for a generic boundary datum. The following example shows that such a maximality property can not be expected in general.

Example 7.4. Let $\Omega:=(0,1) \times(0,1)$ and let $H(x, p):=|p|-a(x), K(x, p):=|p|-b(x)$, where $a(x):=$ $\chi_{\bar{\Omega}}(x)+\chi_{\Omega}(x)$ and $b(x):=2 \chi_{\bar{\Omega}}(x)$. Notice that $S_{K}(x, y)=2|x-y|$ and that $S_{H}=S_{K}$ in a suitable neighborhood of every point of $\Omega$. Let $g(x):=2|x|$ and set, for every $x \in \bar{\Omega}$,

$$
u(x):=\inf _{y \in \partial \Omega}\left\{S_{H}(x, y)+g(y)\right\}, \quad v(x):=\inf _{y \in \partial \Omega}\left\{S_{K}(x, y)+g(y)\right\} .
$$

Notice that $g$ satisfies the compatibility Condition (33) with respect to the Hamiltonian $K$ (but not with respect to $H$ ). In particular, that implies $v=g$ on $\partial \Omega$. By Proposition 5.4, $u$ and $v$ are a Monge solutions (in particular, 
Monge subsolutions) of equation (20) with Hamiltonian $H$ and $K$ respectively. Moreover, since $S_{H}=S_{K}$ locally in $\Omega$ and (24) is a local property, we have that $v$ is a Monge subsolution with respect to $H$ too. Let us show now that $u$ is less than $v$, i.e. that there exists a point $x_{0} \in \bar{\Omega}$ such that $u\left(x_{0}\right)<v\left(x_{0}\right)$. To this aim, take $x_{0}:=(1 / 2,0)$. Indeed, $v\left(x_{0}\right)=g\left(x_{0}\right)=1$, while $u\left(x_{0}\right) \leq S_{H}\left(x_{0}, 0\right)+g(0)=1 / 2$.

We look for conditions sufficient to guarantee the maximality in $\mathcal{S}_{M}$ of the function $u$ defined in (32). A sufficient condition we found is that the optical length function $S$ defined in (22) can be obtained by taking the infimum only over those curves in $\operatorname{Lip}_{x, y}$ which lie in the interior of $\Omega$, possibly except for their endpoints. Note the this condition is not true in general, as can be easily seen by considering $S_{H}$ in Example 7.4.

Theorem 7.5. Let $H$ be an Hamiltonian satisfying $(\mathrm{H})$. If, for every $x, y \in \bar{\Omega}$,

$$
S(x, y)=\inf \left\{\int_{0}^{1} \sigma(\gamma(t), \dot{\gamma}(t)) \mathrm{d} t \mid \gamma \in \operatorname{Lip}_{x, y}, \gamma(t) \in \Omega \text { for all } t \in(0,1)\right\},
$$

then $u$ defined by (32) is maximal in $\mathcal{S}_{M}$.

Proof. Let $\gamma$ be a curve in $\operatorname{Lip}_{x, y}$ such that $\gamma(t) \in \Omega$ for all $t \in(0,1)$ and let $v \in \mathcal{S}_{M}$. For a fixed positive $\delta<1 / 2$, let $\Gamma_{\delta}:=\gamma([\delta, 1-\delta])$. The set $\Gamma_{\delta}$ is compact and contained in $\Omega$, therefore, by Proposition 4.7, we may find a finite partition $\delta=t_{0}<t_{1}<\ldots<t_{m}=1-\delta$ such that $v\left(\gamma\left(t_{i}\right)\right)-v\left(\gamma\left(t_{i+1}\right)\right) \leq S\left(\gamma\left(t_{i}\right), \gamma\left(t_{i+1}\right)\right)$ for each $i$. Therefore

$$
v(\gamma(\delta))-v(\gamma(1-\delta)) \leq \sum_{i=0}^{m-1} S\left(\gamma\left(t_{i}\right), \gamma\left(t_{i+1}\right)\right) \leq \sum_{i=0}^{m-1} \int_{t_{i}}^{t_{i+1}} \sigma(\gamma, \dot{\gamma}) \mathrm{d} t .
$$

By letting $\delta$ go to 0 and by taking the infimum of (37) over all curves $\gamma \in \operatorname{Lip}_{x, y}$ with $\gamma(t) \in \Omega$ for all $t \in(0,1)$, we obtain, in view of Assumption (36) and the continuity of $v$, that

$$
v(x)-v(y) \leq S(x, y) .
$$

In particular the above inequality is true for every $y \in \partial \Omega$, therefore, recalling also that $v \leq g$ on $\partial \Omega$, we have

$$
v(x) \leq \inf _{y \in \partial \Omega}\{S(x, y)+g(y)\}
$$

which gives the claim.

\section{EXAMPLES}

We conclude this paper by discussing some examples. Before going on, we introduce some preliminary notation. Given a closed subset $C$ of $\mathbb{R}^{N}$, we will denote by dist ${ }^{\#}(x, C)$ the signed distance from the set $C$, namely the function defined as follows

$$
\operatorname{dist}^{\#}(x, C):=\operatorname{dist}(x, C)-\operatorname{dist}\left(x, \mathbb{R}^{N} \backslash C\right) \quad \text { for every } x \in \mathbb{R}^{N} .
$$

The dual metric of a Finsler metric $\varphi \in \mathcal{M}$ is the function $\varphi^{*}$ defined by

$$
\varphi^{*}(x, p):=\sup \{\langle p, \xi\rangle \mid \varphi(x, \xi) \leq 1\} \quad \text { for every }(x, p) \in \bar{\Omega} \times \mathbb{R}^{N} .
$$

When the metric $\varphi$ is convex, i.e. $\varphi(x, \cdot)$ is convex for every $x \in \bar{\Omega}$, the following holds (see [9]):

$$
\sup \left\{\langle\xi, p\rangle \mid \varphi^{*}(x, p) \leq 1\right\}=\varphi(x, \xi) \quad \text { for every }(x, \xi) \in \bar{\Omega} \times \mathbb{R}^{N} .
$$


Example 8.1. Let us consider the Hamilton-Jacobi equation

$$
H(x, D u)=0 \quad \text { in } \Omega,
$$

where $H$ satisfies Assumptions (H), and let $S$ be the associated length function. As $S$ is a Finsler distance, it is actually the uniform limit of a sequence of distances $\left(d_{\varphi_{n}}\right)_{n}$, where $\varphi_{n}$ is a continuous Finsler metric belonging $\mathcal{M}$ for each $n \in \mathbb{N}$ (by Th. 4.1 in [11]). For each $n \in \mathbb{N}$, let us set

$$
Z_{n}(x):=\left\{p \in \mathbb{R}^{N} \mid \varphi_{n}^{*}(x,-p) \leq 1\right\} \quad \text { for every } x \in \bar{\Omega},
$$

and $H_{n}(x, p):=\operatorname{dist}^{\#}\left(p, Z_{n}(x)\right)$ for every $(x, p) \in \bar{\Omega} \times \mathbb{R}^{N}$. For each $n \in \mathbb{N}, H_{n}$ is continuous, and it is convex since $Z_{n}(x)$ is a convex set for every $x$. Moreover, if $S_{n}$ is the associated optical length function for each $n \in \mathbb{N}$, then $S_{n}=d_{\varphi_{n}}$ in view of (38) and by definition of optical length function. Therefore, if $g$ is a boundary datum satisfying the compatibility Condition (33) with respect to the length function $S$, the Monge solution $u$ of

$$
\begin{cases}H(x, D v)=0 & \text { in } \Omega \\ v=g & \text { on } \partial \Omega\end{cases}
$$

is the uniform limit of the unique maximal viscosity solutions $u_{n}$ of the problems

$$
\begin{cases}H_{n}(x, D v)=0 & \text { in } \Omega \\ v \leq g & \text { on } \partial \Omega .\end{cases}
$$

Indeed, by the standard theory of viscosity solutions for continuous Hamiltonians, we know that $u_{n}(x)=$ $\inf _{y \in \partial \Omega}\left\{S_{n}(x, y)+g(y)\right\}$ in $\bar{\Omega}$, so the claim easily follows in view of Theorem 5.3 and by the uniform convergence of $S_{n}$ to $S$.

Example 8.2. In equation (39), assume in addition that the Hamiltonian $H$ is such that the associated optical length function $S$ is symmetric, i.e. $S(x, y)=S(y, x)$ for all $x, y \in \bar{\Omega}$ (this happens, for instance, when $H(x, p)$ is even in $p$ ). Then, by [10], Theorem 4.6, there exists a Borel function $a: \bar{\Omega} \rightarrow[\alpha, \beta]$ such that

$$
S(x, y)=\inf \left\{\int_{0}^{1} a(\gamma(t))|\dot{\gamma}(t)| \mathrm{d} t \mid \gamma \in \operatorname{Lip}_{x, y}\right\} \quad \text { for all } x, y \in \bar{\Omega} .
$$

Therefore, with regard to Monge sub and supersolutions, equation (39) is equivalent to the eikonal equation

$$
|D u|=a(x) \quad \text { in } \Omega,
$$

that is, equations (39) and (40) have the same Monge subsolutions and the same Monge supersolutions, since they have the same optical length functions. Moreover, by the density result proven in [10], Theorem 4.3, the continuous Hamiltonians $H_{n}$ of Example 8.1 can be chosen in such a way that $H_{n}(x, p):=|p|-a_{n}(x)$, for a suitable sequence of Borel measurable functions $a_{n}: \bar{\Omega} \rightarrow[\alpha, \beta]$.

Inspired by Example 6.5, we use the same idea to construct an evolutive Hamilton-Jacobi equation with continuous coefficients, for which standard results of the theory of Hamilton-Jacobi equations apply. The Cauchy problem obtained by coupling this equation with a null boundary datum has therefore a unique viscosity solution, which is shown to tend asymptotically to the Monge solution of a stationary Hamilton-Jacobi equation.

Example 8.3. Let $\Omega:=(0,1) \times(-2,2)$ and, for each $t>0$, consider the continuous function $a_{t}: \bar{\Omega} \rightarrow \mathbb{R}$ defined by

$$
a_{t}\left(x_{1}, x_{2}\right):= \begin{cases}1 & \text { if }\left|x_{2}\right| \geq 1 / t \\ 1 / 2+\left|x_{2}\right| t / 2 & \text { otherwise }\end{cases}
$$


Let us define on $\bar{\Omega} \times(0,+\infty)$ a function $a$ by setting $a(x, t):=a_{t}(x)$ for each $t>0$ and $x \in \bar{\Omega}$. We consider the following evolutive Cauchy problem:

$$
\begin{cases}\partial_{t} v(x, t)+|D v|(x, t)=a(x, t) & \text { in } Q:=\Omega \times(0,+\infty) \\ v(x, t)=0 & \text { on } \partial Q\end{cases}
$$

Since $a(x, t)$ is continuous, we know, by the standard theory of Hamilton-Jacobi equations [18], that the above Cauchy problem admits a unique viscosity solution, given by the following formula:

$$
u(x, t):=\inf _{(y, s) \in \partial Q} S((x, t),(y, s)) \quad \text { for all }(x, t) \in Q
$$

where $S$ is the function defined on $\bar{Q} \times \bar{Q}$ as follows:

$$
S((x, t),(y, s)):=\inf \left\{\int_{s}^{t} a(\gamma(\tau), \tau)+H^{*}(\dot{\gamma}(\tau)) \mathrm{d} \tau \mid \gamma \in \operatorname{Lip}_{y, x}([s, t], \bar{\Omega})\right\}
$$

where $\operatorname{Lip}_{y, x}([s, t], \bar{\Omega})$ denotes the space of curves $\gamma \in \operatorname{Lip}([s, t], \bar{\Omega})$ such that $\gamma(s)=y, \gamma(t)=x$. When $s>t$ or $s=t$ and $x \neq y$ this family is empty: in that case we agree that $S((x, t),(y, s))=+\infty$. In the above formula we have denoted by $H^{*}$ the Fenchel transform of $H(p):=|p|$, namely $H^{*}(\xi):=\sup _{p \in \mathbb{R}^{N}}\langle\xi, p\rangle-H(p)$. Notice that, in this case, $H^{*}$ coincides with the indicator function of the closed ball $\bar{B}_{1}(0)$, i.e. $H^{*}(\xi)$ is equal to 0 if $|\xi| \leq 1$ and to $+\infty$ otherwise. In particular, $S$ degenerates outside a cone of vertex $(x, t)$, i.e. $S((x, t),(y, s))=+\infty$ if $t-s<|x-y|$.

We want to study the asymptotic behavior of the solution $u(x, t)$ of $(41)$. Since the functions $a_{t}$ converge pointwise and increasingly on $\bar{\Omega}$, as $t$ tends to $+\infty$, to the discontinuous function $a_{\infty}(x):=\chi_{\bar{\Omega}}(x)-1 / 2 \chi_{\Gamma}(x)$ (where we have denoted by $\Gamma$ the $x_{1}$-axis $\mathbb{R} \times\{0\}$ ), we expect the asymptotic limit of $u(x, t)$ to solve the stationary Hamilton-Jacobi equation

$$
|D v|=a_{\infty}(x) \text { in } \Omega .
$$

In fact, we will show that $u(x, t)$ tends asymptotically, uniformly in $t$, to the Monge solution of the following Dirichlet problem:

$$
\begin{cases}|D v|=a_{\infty}(x) & \text { in } \Omega \\ v=0 & \text { on } \partial \Omega\end{cases}
$$

To this goal, we first recall (see for instance [18], Th. 5.2) that, if in (43) the function $a$ is replaced by a function $b: \bar{\Omega} \rightarrow[\alpha, \beta], 0<\alpha<\beta$ that does not depend on $t$, then, for fixed $(x, t)$ and $(y, s)$ in $\bar{Q}$, we have:

$$
S((x, t),(y, s)) \geq \inf \left\{\int_{0}^{T} b(\gamma(t))|\dot{\gamma}(t)| \mathrm{d} t \mid \gamma \in \operatorname{Lip}_{y, x}([0, T], \bar{\Omega}), T>0\right\}=d_{b}(y, x)
$$

with equality holding if $t-s \geq|y-x| \beta / \alpha$. In particular, by taking into account this remark and using in (43) the fact that $a(x, t) \leq a_{\infty}(x)$ for all $(x, t) \in \bar{Q}$, one easily obtains that $S((x, t),(y, s)) \leq 2 \operatorname{diam}(\Omega) \vee d_{a_{\infty}}(x, y) \leq$ $2 \operatorname{diam}(\Omega)$ for all $(x, t)$ and $(y, s)$ in $\bar{Q}$ such that $S((x, t),(y, s))<+\infty$ (we have denoted by $\operatorname{diam}(\Omega)$ the diameter of the set $\Omega$ ). Let us now fix $(x, t) \in \bar{Q}$ and let $\gamma \in \operatorname{Lip}([s, t], \bar{\Omega}), 0 \leq s<t$, be a minimizing path of (42). Then we have

$$
\frac{1}{2}(t-s) \leq \int_{s}^{t} a(\gamma(\tau), \tau)+H^{*}(\dot{\gamma}(\tau)) \mathrm{d} \tau=u(x, t) \leq 2 \operatorname{diam}(\Omega)
$$

that is $0 \leq t-s \leq r:=4 \operatorname{diam}(\Omega)$. Then, for $t>r$, any path $\gamma \in \operatorname{Lip}([s, t], \bar{\Omega})$, which is minimal for (42), is such that $s \geq t-r>0$, in particular $\gamma(s) \in \partial \Omega$. Therefore, for $t>r$, it is not restrictive to assume that the infimum 
in (42) is taken letting $(y, s)$ vary over the set $\partial \Omega \times[t-r, t]$ only. In particular, as $a_{t-r}(z) \leq a(\tau, z) \leq a_{\infty}(z)$ for every $z \in \bar{\Omega}$ and $s \leq \tau \leq t$, we obtain that

$$
\int_{s}^{t} a_{t-r}(\gamma)+H^{*}(\dot{\gamma}) \mathrm{d} \tau \leq \int_{s}^{t} a(\gamma, \tau)+H^{*}(\dot{\gamma}) \mathrm{d} \tau \leq \int_{s}^{t} a_{\infty}(\gamma)+H^{*}(\dot{\gamma}) \mathrm{d} \tau
$$

Taking the infimum over all possible curves $\gamma$ joining $(y, s) \in \partial \Omega \times[t-r, t]$ to $(x, t)$ and letting $(y, s)$ vary in $\partial \Omega \times[t-r, t]$, by what previously remarked we eventually get

$$
\inf _{y \in \partial \Omega} d_{a_{t-r}}(x, y) \leq u(x, t) \leq \inf _{y \in \partial \Omega} d_{a_{\infty}}(x, y) .
$$

The claim now follows as $a_{t}$ is an increasing sequence of isotropic Riemannian metrics converging pointwise to $a_{\infty}$ on $\bar{\Omega}$ and therefore, by Proposition 2.7, the distance $d_{a_{t}}$ uniformly converges to $d_{a_{\infty}}$ on $\bar{\Omega} \times \bar{\Omega}$ as $t$ goes to $+\infty$. In particular, this easily implies that $u(x, t)$ asymptotically converges, uniformly in $t$, to inf $y \in \partial \Omega d_{a_{\infty}}(x, y)$, which is the Monge solution of (44) (remark that $d_{a_{\infty}}$ is the optical length function associated to the Hamiltonian $\left.H(x, p)=|p|-a_{\infty}(x)\right)$.

The result of the previous example was obtained in a very special case. Nevertheless, with the same idea, one can obtain an analogous result for Monge solutions of eikonal equations of the following form:

$$
\begin{cases}|D v|=a_{\infty}(x) & \text { in } \Omega \\ v=0 & \text { on } \partial \Omega\end{cases}
$$

where $a_{\infty}: \bar{\Omega} \rightarrow[\alpha, \beta]$ is lower or upper semicontinuous and $\alpha$ and $\beta$ are, as usual, fixed positive constants. Indeed, let us assume, for instance, $a_{\infty}$ lower semicontinuous, being the other case analogous. As well known, it is possible to find an increasing sequence of continuous functions $a_{n}: \bar{\Omega} \rightarrow[\alpha, \beta], n \in \mathbb{N}$, such that $a_{\infty}(x)=$ $\sup _{n} a_{n}(x)$ for all $x \in \bar{\Omega}$. Let us define on $\bar{\Omega} \times(0,+\infty)$ a continuous function $a$ by setting $a(x, t):=(n+1-$ $t) a_{n}(x)+(t-n) a_{n+1}(x)$ for all $x \in \bar{\Omega}, t \in(n, n+1]$ and $n \in \mathbb{N}$. Arguing as above, one immediately gets that the viscosity solution of

$$
\begin{cases}\partial_{t} v(x, t)+|D v|(x, t)=a(x, t) & \text { in } Q:=\Omega \times(0,+\infty) \\ v(x, t)=0 & \text { on } \partial Q\end{cases}
$$

tends asymptotically to the Monge solution of (46).

Acknowledgements. The authors wish to thank Giuseppe Buttazzo for having addressed their attention to this problem and for many useful discussions on the subject. The authors also wish to thank for helpful hints Giovanni Alberti, Fabio Camilli and Pietro Majer. Last, the authors express their gratitude to the Referee for having suggested how to simplify the proof of the comparison theorem.

\section{REFERENCES}

[1] L. Ambrosio and P. Tilli, Selected topics on "Analysis on Metric spaces". Scuola Normale Superiore di Pisa (2000).

[2] M. Bardi and I. Capuzzo Dolcetta, Optimal control and viscosity solutions of Hamilton-Jacobi-Bellman equations. Syst. Control Found. Appl. (1997).

[3] G. Barles, Solutions de viscosité des équations de Hamilton-Jacobi. Math. Appl. 17 (1994).

[4] E.N. Barron and R. Jensen, Semicontinuous viscosity solutions for Hamilton-Jacobi equations with convex Hamiltonians. Comm. Partial Diff. Equ. 15 (1990) 1713-1742.

[5] G. Buttazzo, Semicontinuity, relaxation and integral representation in the calculus of variations. Pitman Res. Notes Math. Ser. 207 (1989).

[6] G. Buttazzo, L. De Pascale and I. Fragalà, Topological equivalence of some variational problems involving distances. Discrete Contin. Dyn. Syst. 7 (2001) 247-258. 
[7] L. Caffarelli, M.G. Crandall, M. Kocan and A. Swiech, On viscosity solutions of fully nonlinear equations with measurable ingredients. Comm. Pure Appl. Math. 49 (1996) 365-397.

[8] F. Camilli and A. Siconolfi, Hamilton-Jacobi equations with measurable dependence on the state variable. Adv. Differ. Equ. 8 (2003) 733-768.

[9] F.H. Clarke, Optimization and Nonsmooth Analysis. John Wiley \& Sons, New York (1983).

[10] A. Davini, On the relaxation of a class of functionals defined on Riemannian distances. J. Convex Anal., to appear.

[11] A. Davini, Smooth approximation of weak Finsler metrics. Adv. Differ. Equ., to appear.

[12] G. De Cecco and G. Palmieri, Length of curves on LIP manifolds. Rend. Accad. Naz. Lincei, Ser. 91 (1990) $215-221$.

[13] G. De Cecco and G. Palmieri, Integral distance on a Lipschitz Riemannian Manifold. Math. Z. 207 (1991) 223-243.

[14] G. De Cecco and G. Palmieri, Distanza intrinseca su una varietà finsleriana di Lipschitz. Rend. Accad. Naz. Sci. V, XVII, XL, Mem. Mat. 1 (1993) 129-151.

[15] G. De Cecco and G. Palmieri, LIP manifolds: from metric to Finslerian structure. Math. Z. 218 (1995) $223-237$.

[16] H. Ishii, A boundary value problem of the Dirichlet type for Hamilton-Jacobi equations. Ann. Sc. Norm. Sup. Pisa 16 (1989) $105-135$.

[17] H. Ishii, Hamilton-Jacobi equations with discontinuous Hamiltonians on arbitrary open sets. Bull. Facul. Sci. E Eng., Chuo Univ., Ser I 28 (1985) 33-77.

[18] P.L. Lions, Generalized solutions of Hamilton Jacobi equations. Pitman (Advanced Publishing Program). Res. Notes Math. 69 (1982).

[19] R.T. Newcomb II and J. Su, Eikonal equations with discontinuities. Differ. Integral Equ. 8 (1995) 1947-1960.

[20] P. Soravia, Boundary value problems for Hamilton-Jacobi equations with discontinuous Lagrangian. Indiana Univ. Math. J. 51 (2002) 451-477. 